\title{
Stabilization of the extended horizons
}

\author{
Alex Buchelø \\ Department of Applied Mathematics Department of Physics \\ and Astronomy University of Western Ontario London, Ontario N6A 5B7, Canada \\ and Perimeter Institute for Theoretical Physics Waterloo, Ontario N2J 2W9, Canada
}

(Received 1 June 2021; accepted 30 July 2021; published 26 August 2021)

\begin{abstract}
We extend Kodama-Ishibashi and Jansen-Rostworowski-Rutkowski master field framework to study quasinormal modes of black branes and black holes of Einstein gravity in $D=5$ space-time dimensions with multiple scalars and an arbitrary bulk potential. As an application, we consider a thermodynamically unstable state of mass deformed $\mathcal{N}=4$ supersymmetric Yang-Mills theory at strong coupling. The corresponding gravitational dual is $\mathcal{N}=2^{*}$ black brane with a dynamical instability of its translationary invariant horizon. We study the dependence of the quasinormal mode spectra as the boundary gauge theory is compactified on $S^{3}$-the black brane is turned into the black hole.
\end{abstract}

DOI: 10.1103/PhysRevD.104.046025

\section{INTRODUCTION}

One of the thermodynamic inequalities of a thermal state at equilibrium, also known as a condition for a thermodynamic stability, is [1],

$$
\frac{c_{v}}{s} \equiv \frac{T}{s}\left(\frac{\partial s}{\partial T}\right)_{v}>0,
$$

where $T$ is a temperature density, $s$ is an entropy density and $c_{v}$ is a constant volume specific heat density. In gauge theory/string theory correspondence [2,3], the properties of thermal states of strongly coupled gauge theories are encoded in thermodynamics of the corresponding planar black hole horizon geometries. The thermodynamic stability is equally applicable to black branes: here, the entropy density is the Bekenstein entropy density of a regular Schwarzschild planar horizon, and the temperature is its Hawking temperature. Perturbed black branes relax to thermal equilibrium via quasinormal modes (QNMs) [4]. In some cases the spectra of QNMs contains unstable states, i.e., the QNMs with ${ }^{1}$

$$
\operatorname{Im}[\mathfrak{w}]>0 .
$$

When excited, these modes grown exponentially (in a linearized approximation), rather then decaying. In the dual

\footnotetext{
${ }^{1}$ We define reduced frequency and momenta as $\mathfrak{w} \equiv w /(2 \pi T)$ and $\mathfrak{q}=k /(2 \pi T)$.

Published by the American Physical Society under the terms of the Creative Commons Attribution 4.0 International license. Further distribution of this work must maintain attribution to the author(s) and the published article's title, journal citation, and DOI. Funded by SCOAP ${ }^{3}$.
}

boundary language, their presence signals a dynamical instability of the strongly coupled plasma to densitypressure fluctuations breaking the translational invariance of the thermal equilibrium state.

In $[5,6]$ the authors formulated the correlated stability conjecture (CSC): A black brane thermodynamic (in) stability correlates with its dynamical (in)stability The conjecture is applicable only to horizons with translational invariance, e.g., simple higher-dimensional Schwarzschild black holes in Einstein gravity with a cosmological constant are stable [7]. If true, the CSC would provide a way to bypath technically complicated QNM analysis and reestablish the dynamical (in)stability of a horizon.

In one direction, the CSC is trivial [8]: thermodynamic instability of an extended horizon implies its dynamical instability. Indeed, a basic thermodynamic relation between the speed of the sound waves in (uncharged) plasma $c_{s}$ and its specific heat

$$
c_{s}^{2}=\frac{s}{c_{v}}
$$

implies that when $c_{v}<0$ the speed of the sound waves is purely imaginary. As a result, the sound mode, i.e., the scalar channel black brane QNM,

$$
\mathfrak{w}(\mathfrak{q})= \pm c_{s} \mathfrak{q}-2 \pi i \frac{\eta}{s}\left(\frac{2}{3}+\frac{\zeta}{2 \eta}\right) \mathfrak{q}^{2}+\mathcal{O}\left(\mathfrak{q}^{3}\right)
$$

is unstable for some sign and for small enough q. In (1.4), $\eta$ and $\zeta$ are the shear and the bulk viscosities of the plasma. The presence of such dynamical instability in thermodynamically unstable horizons was explicitly verified in [8-11]. 
In the other direction the conjecture is simply wrong. ${ }^{2} \mathrm{~A}$ sharp recent counterexample is the holographic conformal order [13-15]. It is straightforward to construct holographic models in asymptotically $\operatorname{AdS}_{d+2}$ with, say $\mathbb{Z}_{2}$ global symmetry, such that

$\frac{\mathcal{F}}{T^{d+1}}=-\mathcal{C} \times \begin{cases}1, & \langle\mathcal{O}\rangle=0 \Rightarrow \mathbb{Z}_{2} \text { is unbroken; } \\ \kappa, & \langle\mathcal{O}\rangle \neq 0 \Rightarrow \mathbb{Z}_{2} \text { is spontanuously broken }\end{cases}$

where $\mathcal{F}$ is the free energy density, $\mathcal{C}$ is a positive constant proportional to the central charge of the theory, and $0<\kappa<1$ is also a constant. The thermodynamics and the hydrodynamics of both the symmetric and the symmetry broken phases are identical, i.e., that of the $\mathrm{CFT}_{d+1}$. In particular, see (1.1),

$$
\frac{c_{v}}{s}=d>0,
$$

i.e., both phases are thermodynamically stable. On the other hand, there is [15] a (nonhydrodynamic) branch of the scalar sector QNMs that renders the symmetry broken phase perturbatively unstable, i.e., there is a QNM of the type (1.2) at $\mathfrak{q}=0$.

The motivation of this study is to "fix" the instabilities of the extended horizons, by compactifying the Euclidean space $\mathbb{R}^{d}$ of a dual holographic $\mathrm{CFT}_{d+1}\left(\mathrm{QFT}_{d+1}\right.$ more generally) on $d$-dimensional round sphere $S^{d}$ :

$$
\mathbb{R}^{d} \rightarrow L^{2} S^{d}, \quad K \equiv \frac{1}{L^{2}} .
$$

Specific potential applications are

(i) Curing the instabilities in the hydrodynamic sector, i.e., in models with $c_{s}^{2}<0$. An example: KlebanovStrassler black branes [16].

(ii) Curing the instabilities in the nonhydrodynamic sector, i.e., in models of the holographic conformal order [15].

The rest of the paper is organized as follows. In Appendix A we extend the QNM analysis of $[17,18]$ to black branes/holes in theories of Einstein gravity in $D=5$ space-time dimensions with multiple scalars and an arbitrary bulk potential:

$S_{5}=\int_{\mathcal{M}_{5}} d^{5} \xi \sqrt{-g}\left[R-\sum_{j=1}^{p} \eta_{j}\left(\partial \phi_{j}\right)^{2}-V\left(\left\{\phi_{j}\right\}\right)\right]$.

We then apply the general formalism to $\mathcal{N}=2^{*}$ holographic model [19-21], summarizing the results in Sec. II. Technical details of the computations are discussed in

\footnotetext{
${ }^{2}$ See $[11,12]$ for an earlier work.
}

Sec. III. We conclude in Sec. IV. Summary of the numerical tests performed is presented in Appendix B.

Thermodynamically unstable phase of $\mathcal{N}=2^{*}$ plasma was identified in [22]. The hydrodynamics of this phase, and its dynamical instability, was discussed in $[9,23,24]$. Detailed analysis of the $S^{3}$-compactified $\mathcal{N}=2^{*}$ unstable thermodynamics is new. ${ }^{3}$ Computation of the QNM spectra of $\mathcal{N}=2^{*}$ black holes is new.

\section{SUMMARY}

In this section we present results ${ }^{4}$ of the case study of the extended unstable horizons in $\mathcal{N}=2^{*}$ holographic model. We focus on a particular top-down holographic model, and it is interesting to explore in the future how generic the reported results are. It is a model of a real string holography $[20,28-30]$ — we feel it is important to emphasize this as sometimes subtle effects in toy holographic models do not occur in string theory $[14,31]$.

We focus on the thermodynamically unstable phase of the $\mathcal{N}=2^{*}$ theory, where a single mass parameter $m$ is introduced to the bosonic components of the $\mathcal{N}=2$ hypermultiplet of $\mathcal{N}=4$ supersymmetric $S U(N)$ YangMill theory. We work in the planar limit of the gauge theory, and at large 't Hooft coupling - in this case the full string theory/gauge theory duality is reduced to a supergravity approximation [2]. The full ten dimensional type IIB supergravity can be consistently truncated on $S^{5}$, producing the Pilch-Warner (PW) effective action [19]. The latter effective action is in the general class of models covered in Appendix A.

The general plan is as follows.

(1) We begin with the $\mathcal{N}=2^{*}$ black brane thermodynamics and hydrodynamics.

(a) We identify a thermodynamically unstable state of $\mathcal{N}=2^{*}$ model at $K=0$ - this is a purple $\operatorname{dot}^{5}$ in Fig. 1, and the left panel of Fig. 3.

(b) Next, we compute the hydrodynamic properties of this state-Fig. 4. We establish that the speed of the sound waves $c_{s}^{2}<0$, and compute the bulk viscosity $\zeta$ (the purple dots, both panels). We use the hydrodynamic limit (1.4) as an independent check on the computation of the QNM spectra in the framework of Appendix A-the thin red dashed curves in Fig. 5.

(c) The sound channel QNM of the selected state (the purple dot) is computed in Fig. 5. We establish the expected [8] dynamical instability.

(2) We continue with the nonhydrodynamic QNMs of the $\mathcal{N}=2^{*}$ black brane.

\footnotetext{
${ }^{3}$ Some early work appeared in $[25,26]$.

${ }^{4}$ Also available as a recent talk [27].

${ }^{5}$ There is nothing special with our selection of the thermodynamically unstable state - any state on the red dashed curves would do. The results are qualitatively the same.
} 

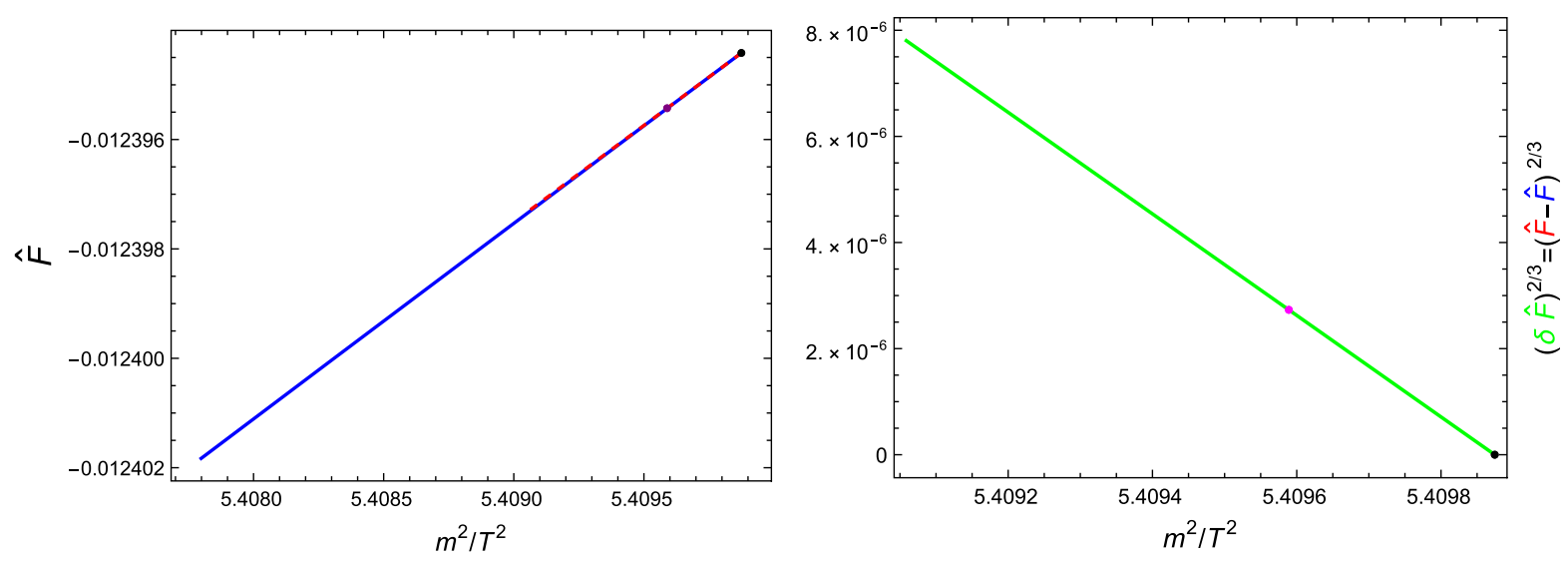

FIG. 1. Thermodynamics of $\mathcal{N}=2^{*}$ model in the canonical ensemble at $K=0$. The solid blue curve is the thermodynamically stable phase with $c_{v}>0$; the dashed red curve is the thermodynamically unstable phase with $c_{v}<0$. Both phases join at the terminal temperature, denoted by the black dot. The stable phase has a lower free energy density, and is the thermodynamically preferred one (right panel). The purple dot indicates the thermal state (in the thermodynamically unstable phase) were we compute the QNM spectra and follow them as $K \neq 0$.

(a) We compute the spectrum of the nonhydrodynamic modes in the helicity $h=0$ sector at $\mathfrak{q}=0$, initially at $m^{2}=0$, i.e., in pure $\mathrm{AdS}_{5^{-}}$ Schwarzschild black brane, see Fig. 6. This is one of the two branches of the QNMs in this helicity sector - the other branch contains the hydrodynamic (sound) mode with $\mathfrak{w}(\mathfrak{q}=0)=0$, detailed in Fig. 5. The two branches decouple at $\mathfrak{q}=0$. We highlight the two lowest QNMs: the magenta and the pink one, which we follow, increasing $\frac{m^{2}}{T^{2}}$, to the select unstable thermal state of interest at (2.14).

(b) The dependence of the magenta (the lowest in Fig. 6) QNMs at $\mathfrak{q}=0$ as $\frac{m^{2}}{T^{2}}$ varies as

$$
0 \leq \frac{m^{2}}{T^{2}} \leq \frac{m^{2}}{T_{p}^{2}}
$$

is shown in Fig. 7. Figure 8 tracks over the same mass range the pink QNMs in Fig. 6. Both modes remain stable, i.e., have $\operatorname{Im}[\mathfrak{w}]<0$, over the full mass range (2.1).

(3) We proceed introducing the curvature to the black brane horizon of the select unstable state. We omit the description of the background, and present only the results for the QNMs at temperature (2.14) as $\frac{K}{m^{2}}$ varies. A sample background thermodynamics at $\frac{K}{m^{2}}=1$ is shown in Fig. 2 and the right panel of Fig. 3.

In the absence of curvature, the dispersion of the low energy quasinormal modes is characterized by few transport coefficients (the speed of the sound waves $c_{s}$, the shear $\eta$ and the bulk $\zeta$ viscosities) —see (1.4) and Fig. 5-valid, provided

$$
\mathfrak{q} \equiv \frac{k}{2 \pi T} \ll 1 \quad \text { and } \quad \mathfrak{q} \cdot \frac{T}{m} \ll 1 .
$$

The finite curvature $\frac{K}{m^{2}} \neq 0$ does not invalidate the hydrodynamics, but simply restricts its applicability according to (2.2). Indeed, compactification of the theory on $S^{3}$ 'quantizes' the available spatial momenta according to (A11)

$$
\mathfrak{q}^{2}=\frac{K \ell(\ell+2)}{4 \pi^{2} T^{2}}, \quad \ell \in \mathbb{Z}_{+},
$$

which constrains the order of the harmonic $\ell$ well described by (1.4). For example, for $K \ll \min \left\{T^{2}, m^{2}\right\}$,

$$
2 \leq\left.\ell\right|_{\text {hydro }} \ll \ell_{\max } \equiv \min \left\{\frac{2 \pi T}{\sqrt{K}}, \frac{2 \pi m}{\sqrt{K}}\right\} .
$$

Note the lower bound on $\ell$ : the hydrodynamic fluctuation are fluctuations of the energy density/pressure, which are physical on $S^{3}$ only for $\ell \geq 2$, see Appendix A. Of course, one can improve the agreement of the exact QNM dispersion $\mathfrak{w}(\mathfrak{q})$ with its hydrodynamic approximation (1.4) by including the higher-order hydrodynamic transport coefficients, specifically the second-order curvature coupling coefficient $\kappa$ [32]. In this paper we compute $\mathfrak{w}(\mathfrak{q})$ for generic $\mathfrak{q}$, without relying on hydrodynamic approximations: $\ell=0$ and $\ell=1$ instabilities (see Fig. 9) are always outside the hydrodynamic approximation; likewise are outside the hydrodynamic approximation all $\ell=2$ branches in Fig. 13 except for the black and the brown subbranches for $\frac{K}{m^{2}} \ll 1$.

(i) As discussed in Appendix A, QNMs of the black holes with spherical horizon at $\ell=\{0,1\}$ are physically distinct from those with $\ell \geq 2$ : the former ones include physical fluctuations exclusively in the 

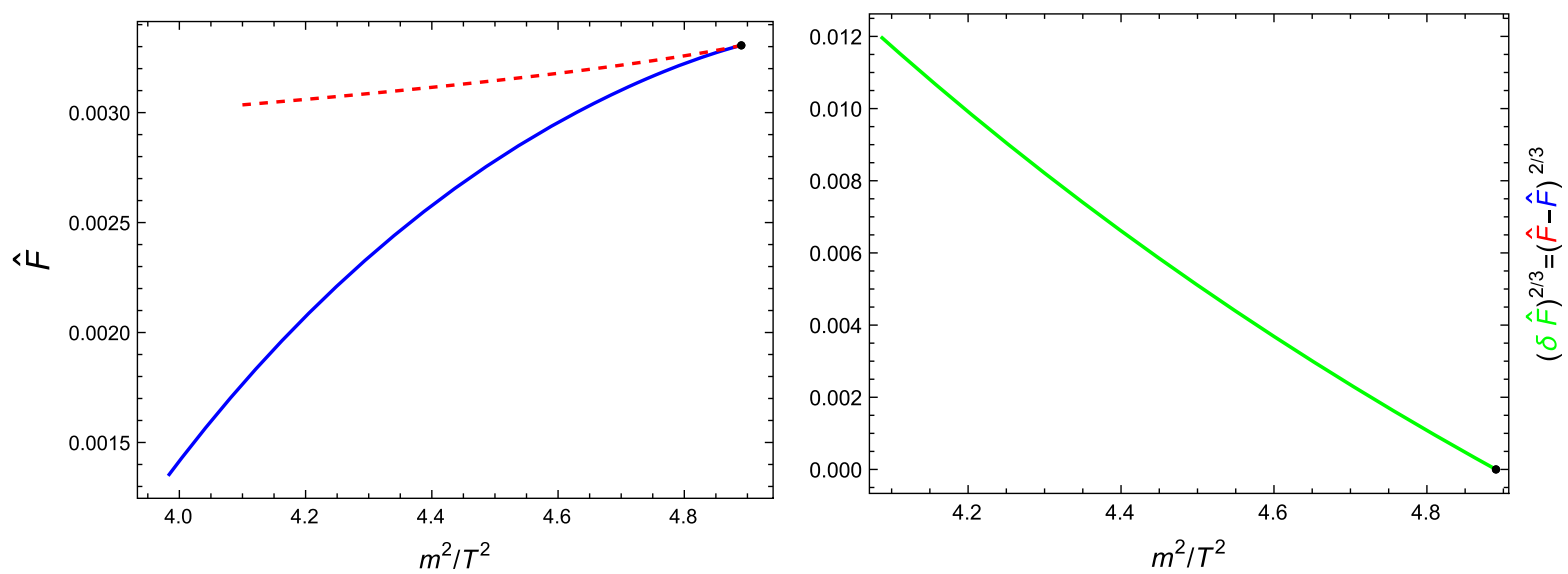

FIG. 2. Thermodynamics of $\mathcal{N}=2^{*}$ model in the canonical ensemble at $K=m^{2}$ is qualitatively the same as for $K=0$, see Fig. 1 .
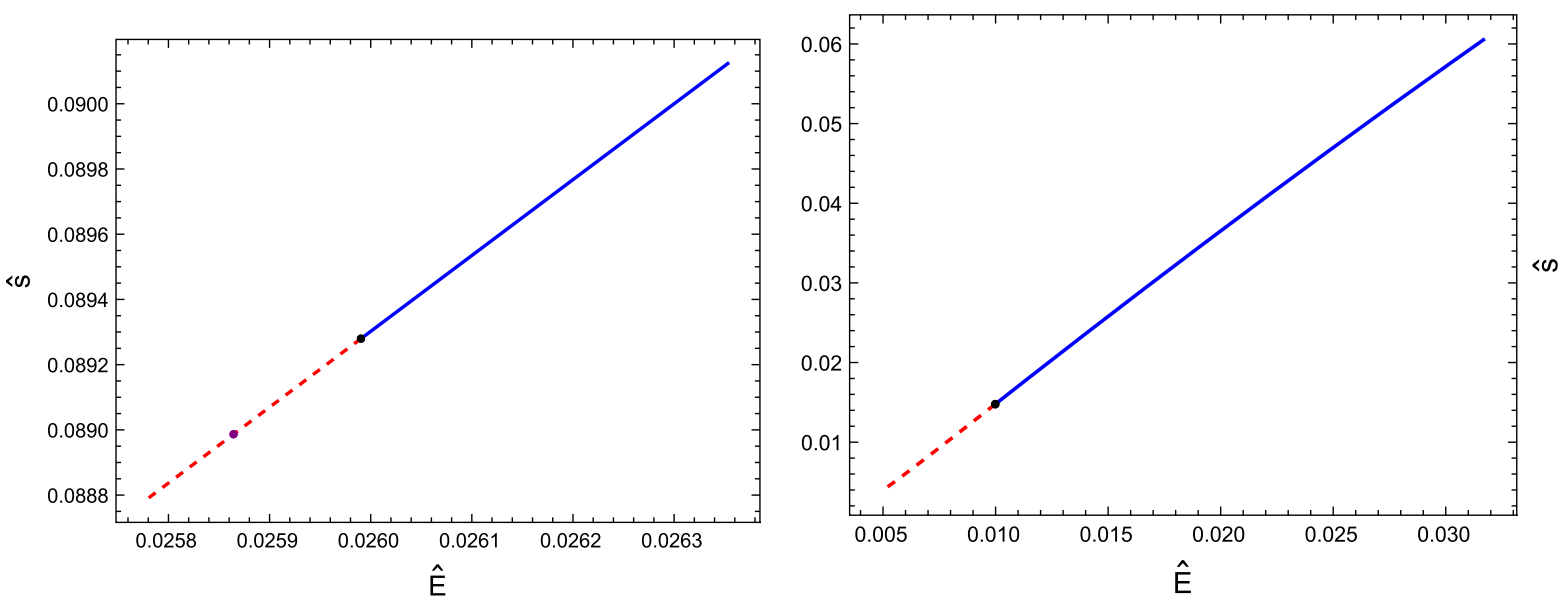

FIG. 3. Thermodynamics of $\mathcal{N}=2^{*}$ model in the microcanonical ensemble at $K=0$ (the left panel) and $K=m^{2}$ (the right panel). The thermodynamically stable phases (the solid blue curves) terminate at the $\hat{\mathcal{E}}_{\text {cit }}$, denoted by the black dot. Thermal states with $\hat{\mathcal{E}}<\hat{\mathcal{E}}_{\text {crit }}$ are thermodynamically unstable (the red dashed curves). The purple dot indicates the reduced energy density $\hat{\mathcal{E}}_{p}$ of the state selected for the QNM spectra analysis.

bulk gravitational scalar sector; while the latter ones mix the bulk scalar and the physical metric fluctuations. In Fig. 9 we present the dispersion of $\ell=0$ (solid curves) and $\ell=1$ (dashed curves) QNMs with $\frac{K}{m^{2}}$ at fixed temperature (2.14). At $K=0$ these modes start from the magenta QNMs of Fig. 7. The $\ell=1$ mode is stable. While the $\ell=0$ mode starts as the stable ones at $K=0$, it develops an instability in the range

$$
\frac{K_{\ell=0}^{\text {stable }}}{m^{2}} \leq \frac{K}{m^{2}} \leq \frac{K_{\ell=0}^{\text {unstable }}}{m^{2}} .
$$

Of course, the starting point at $K=0$ can be any $\mathrm{AdS}_{5}$ QNM of Fig. 6 evolved to the required value of $\frac{m^{2}}{T_{p}^{2}}$, see (2.14)-e.g., in Fig. 11 we include the $\frac{K}{m^{2}}$ dispersion of the $\ell=0$ and $\ell=1$ modes starting from the pink QNMs of Fig. 8. (ii) In Fig. 13 we present the dispersion of $\ell=2$ QNMs with $\frac{K}{m^{2}}$ at fixed temperature (2.14). We discuss only the modes that originate at $K=0$ from the magenta QNMs of Fig. 7, and the hydrodynamic (sound) mode (the cyan dot with $\operatorname{Im}[\mathfrak{w}]=0$ ). The $\ell=2$ mode remains unstable from $K=0$ up to $K_{\ell=2}^{\text {unstable }}$,

$$
0 \leq \frac{K}{m^{2}} \leq \frac{K_{\ell=2}^{\text {unstable }}}{m^{2}}
$$

(iii) Interestingly, $K_{\ell=2}^{\text {unstable }}<K_{\ell=0}^{\text {stable }}$, thus there is stability range of $\mathcal{N}=2^{*}$ black holes at the intermediate values of $K$ :

$$
\frac{K_{\ell=2}^{\text {unstable }}}{m^{2}}<\frac{K}{m^{2}}<\frac{K_{\ell=0}^{\text {stable }}}{m^{2}} .
$$


(iv) Finally, in Fig. 14 we present the dispersion of higher- $\ell$ QNMs with $\frac{K}{m^{2}}$ at fixed temperature (2.14). We highlight only the unstable subbranch of the QNMs, the one that originates at $K=0$ from the hydrodynamic (sound) mode. All these modes are unstable similar to $\ell=2$ mode, see (2.6), with distinct $K_{\ell}^{\text {unstable }}$ such that

$$
K_{\ell}^{\text {unstable }}>K_{\ell^{\prime}}^{\text {unstable }}, \quad \text { if } \ell^{\prime}>\ell .
$$

In the following plots we always present dimensionless quantities. Dimensionless frequency and momenta of the QNMs are defined as in footnote 1 . We further introduce the reduced free energy density $\hat{\mathcal{F}}$, the reduced energy density $\hat{\mathcal{E}}$, and the reduced entropy density $\hat{s}$ as follows:

$\hat{\mathcal{F}}=\frac{8}{3 \pi^{2} N^{2}} \frac{\mathcal{F}}{m^{4}}, \quad \hat{\mathcal{E}}=\frac{8}{3 \pi^{2} N^{2}} \frac{\mathcal{E}}{m^{4}}, \quad \hat{s}=\frac{8}{3 \pi^{2} N^{2}} \frac{s}{m^{3}}$,

where the overall prefactor is chosen in such a way that

$$
\lim _{m \rightarrow 0}\left[\frac{m^{4}}{T^{4}} \hat{\mathcal{E}}\right]=1
$$

\section{A. The thermodynamics and the hydrodynamics of the model at $K=0$}

In Fig. 1 we present the thermodynamics of the model at $K=0$ in the canonical ensemble [33]. There is a critical (terminal) temperature $T_{\text {crit }, 0}$ (denoted by the black dot)

$$
\frac{m^{2}}{T_{\text {crit }, 0}^{2}}=5.4098(7)
$$

where the two phases join: the solid blue curve is the phase with $c_{v}>0$ while the red dashed curve represents the phase with $c_{v}<0$. As $T \rightarrow T_{\text {crit }, 0}$ (from above) the specific heat diverges as

$$
\frac{c_{v}}{S} \propto \pm\left(1-\frac{T_{\text {crit, } 0}}{T}\right)^{-1 / 2}
$$

The free energy density of the thermodynamically unstable phase is always above that of the stable phase. This is more clear in the right panel, were we plot

$$
\begin{aligned}
(\delta \hat{\mathcal{F}})^{2 / 3} & \equiv(\hat{\mathcal{F}}-\hat{\mathcal{F}})^{2 / 3} \\
& \propto+\left(1-\frac{T_{\text {crit }, 0}}{T}\right), \quad \text { as } T \rightarrow T_{\text {crit }, 0} .
\end{aligned}
$$

The linear scaling of $(\delta \hat{\mathcal{F}})^{2 / 3}$ with the temperature near the criticality is a reflection of (2.12). Note the purple dot in the red (thermodynamically unstable phase)—more pronounced in the right panel-at temperature $T_{p}$,

$$
\frac{m^{2}}{T_{p}^{2}}=5.40959
$$

We will study the QNM spectra of the model for different $\frac{K}{m^{2}}$, keeping the temperature fixed as in (2.14).

As shown in Fig. 2, the canonical ensemble phase diagram is qualitatively unchanged as $K \neq 0$; here $K=m^{2}$. While the critical temperature at $\frac{K}{m^{2}}=1, T_{\text {crit }, 1}$

$$
T_{\text {crit }, 1}>T_{\text {crit }, 0},
$$

we find that $T_{\text {crit }}$ is not a monotonically increasing function of $\frac{K}{m^{2}}$, e.g.,

$$
\begin{aligned}
\left.\frac{m^{2}}{T_{\text {crit }}}\right|_{K / m^{2}=0.628169} & =7.266(0)>\left.\frac{m^{2}}{T_{\text {crit }}}\right|_{K / m^{2}=0}>\left.\frac{m^{2}}{T_{\text {crit }}}\right|_{K / m^{2}=1} \\
& =4.8903(1) .
\end{aligned}
$$

In Fig. 3 we present the thermodynamics of the model in the microcanonical ensemble: $K=0$ (the left panel) and $K=m^{2}$ (the right panel). The black dots indicate the critical point of the canonical ensemble at $\frac{K}{m^{2}}=0$ and $\frac{K}{m^{2}}=1$,

$\hat{\mathcal{E}}_{\text {crit }, 0}=0.025990(4), \quad \hat{\mathcal{E}}_{\text {crit }, 1}=0.0099855(1)$.

The color coding is the same as in Figs. 1 and 2. The purple dot (the left panel) identifies the state which we use to study the QNM spectra of the $\mathcal{N}=2^{*}$ thermodynamically unstable horizon,

$$
\hat{\mathcal{E}}_{p}=0.0258644
$$

In Fig. 4 we present the results for the speed of the sound waves [22] (the left panel), and the bulk viscosity (the right panel) of the $\mathcal{N}=2^{*}$ black branes $[9,23,34]$. For consistency, we use the same color coding as in earlier plots: the solid blue curves denote the thermodynamically stable phase, and the red dashed ones represent the thermodynamically unstable phase. As expected from [8], $c_{s}^{2}<0$ in the thermodynamically unstable phase. Note that the speed of the sound waves vanishes at $T_{\text {crit, },}$, see (2.11), responsible [according to (1.3)] for the divergence of the specific heat (2.12). The bulk viscosity is finite at the criticality, denoted as the black dot. The unstable thermal state of interest once again is indicated with the purple dot-here the temperature $T_{p}$ is given by (2.14), and the specific values of the transport coefficients are

$c_{s}^{2}=-0.016312(8), \quad \frac{\zeta}{\eta}=7.1791(6), \quad \frac{\eta}{s}=\frac{1}{4 \pi}$, 

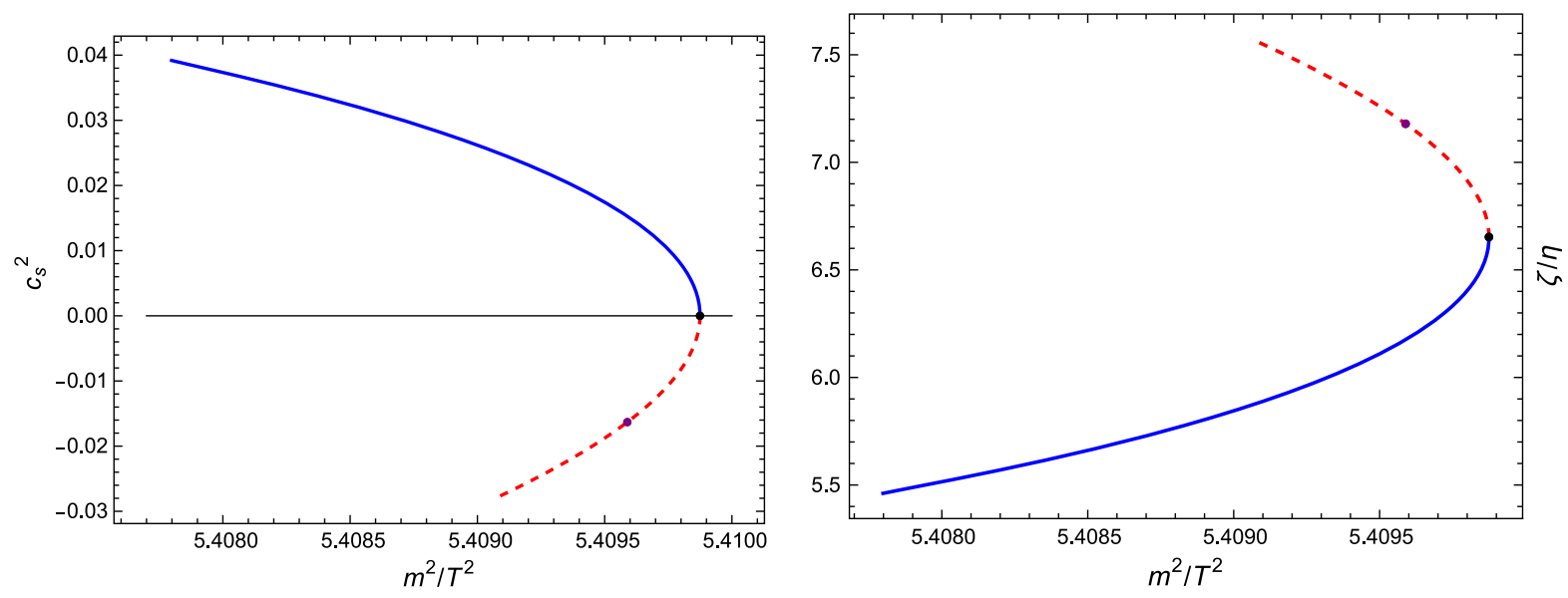

FIG. 4. The speed of the sound waves $c_{s}^{2}$ (the left panel) and the ratio of bulk-to-shear viscosities $\frac{\zeta}{\eta}$ (the right panel) in $\mathcal{N}=2^{*}$ black branes close to a critical point (2.11) (the black dot). Thermodynamically stable and unstable phases are represented by the solid and the dashed curves correspondingly. The purple dot identifies the thermal state of interest, see (2.19) for the values of its transport coefficients.

where we included the universal result for the shear viscosity $\eta$ as well $[35,36]$. The knowledge of the transport (2.19) provides a prediction for the dispersion of the hydrodynamic sound waves as in (1.4), and a valuable test of the QNM computations reported in Fig. 5 in the framework of Appendix A.

In Fig. 5 we present the dispersion $\mathfrak{w}=\mathfrak{w}(\mathfrak{q})$ of the hydrodynamic quasinormal mode of the $\mathcal{N}=2^{*}$ black branes in the thermodynamically unstable state represented by the purple dot in Figs. 1, 3 and 4, at temperature (2.14), and the energy density (2.18). The dashed red curves represent the $\mathcal{O}\left(\mathfrak{q}^{2}\right)$ hydrodynamic approximation (1.4), with the appropriate transport coefficients (2.19). The solid black and blue curves represent $\mathfrak{w}(\mathfrak{q})$ for the unstable and the stable subbranches of the hydrodynamic QNM computed in the framework of Appendix A for $\mathfrak{q} \geq \frac{1}{50}$. Note the

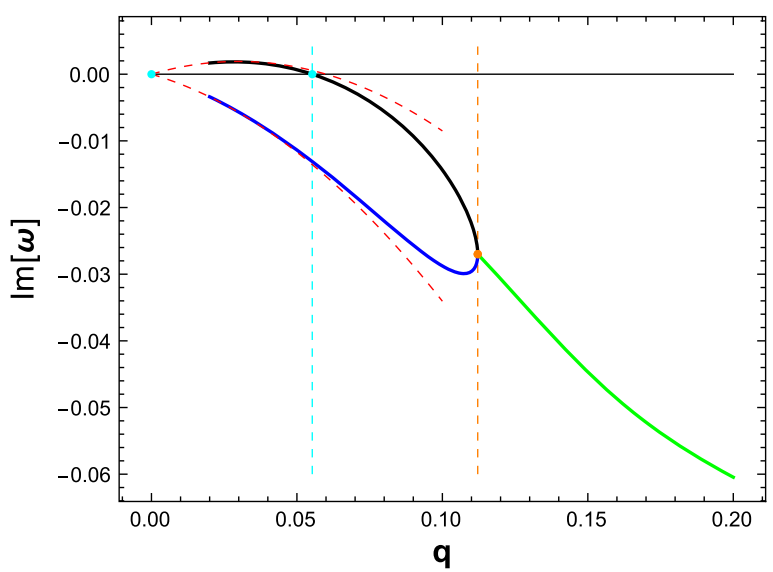

agreement with the hydrodynamic predictions (1.4) for small $\mathfrak{q}$. The black/blue subbranches are purely imaginary, and coalesce at

$$
\mathfrak{q}_{o}=0.11215(1),
$$

represented by the vertical dashed orange lines. For $\mathfrak{q}>\mathfrak{q}_{o}$ the hydrodynamic sound mode, nonpropagating for $\mathfrak{q} \in\left[0, \mathfrak{q}_{o}\right]$, becomes propagating, i.e., it develops $\operatorname{Re}[\mathfrak{w}] \neq 0$. The propagating part of the sound hydrodynamic QNM is represented with solid green curves. As expected from the general arguments [8], the $\mathcal{N}=2^{*}$ thermodynamically unstable black brane state (2.14) is dynamically unstable for

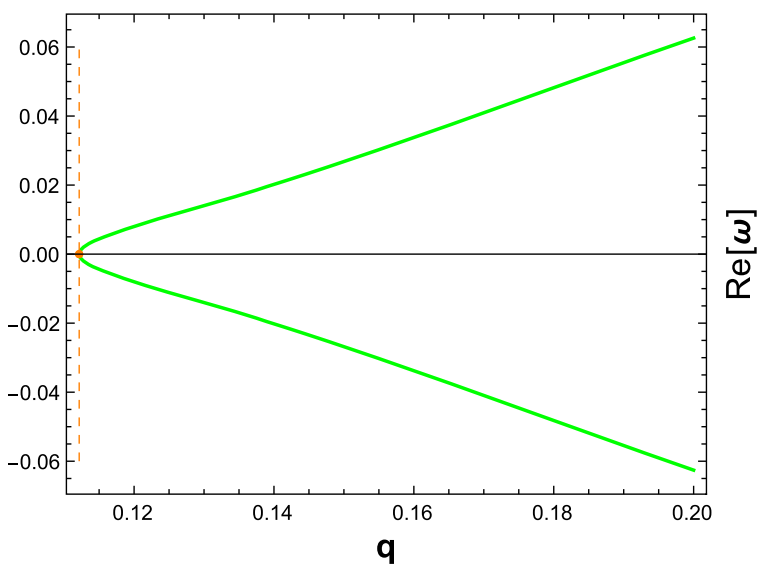

FIG. 5. The dispersion relation of the hydrodynamic QNM $\mathfrak{w}(\mathfrak{q})$ of the thermodynamically unstable state of the $\mathcal{N}=2^{*}$ black brane at temperature (2.14). The dashed red curves represent the $\mathcal{O}\left(\mathfrak{q}^{2}\right)$ hydrodynamic approximation (1.4). The mode is nonpropagating for $\mathfrak{q}<\mathfrak{q}_{o}$, represented by the vertical dashed orange lines. It is unstable (the black branch) in the range of $\mathfrak{q}$ between the two cyan dots, see (2.21). 


$$
\mathfrak{q} \in\left(0, \mathfrak{q}_{\text {unstable }}\right), \quad \mathfrak{q}_{\text {unstable }}=0.055273(8),
$$

represented by the vertical dashed cyan line.

\section{B. Nonhydrodynamic QNMs of the model at $K=0$}

We begin discussion of the nonhydrodynamic QNMs of the $\mathcal{N}=2^{*}$ black brane at $m=0$, i.e., in the pure $\mathrm{AdS}_{5}$-Schwarzschild limit in Fig. 6. There are two distinct $h=0$ subsectors, which are decoupled when $m=0$. One of these subsectors contains a hydrodynamic sound mode; we focus on the other subsector at $\mathfrak{q}=0$. We highlight the two lowest pairs of the QNMs: the magenta and the pink one. In Fig. 7 we follow the magenta QNMs, increasing $m$ to reach the thermodynamically unstable state of interest at temperature (2.14):

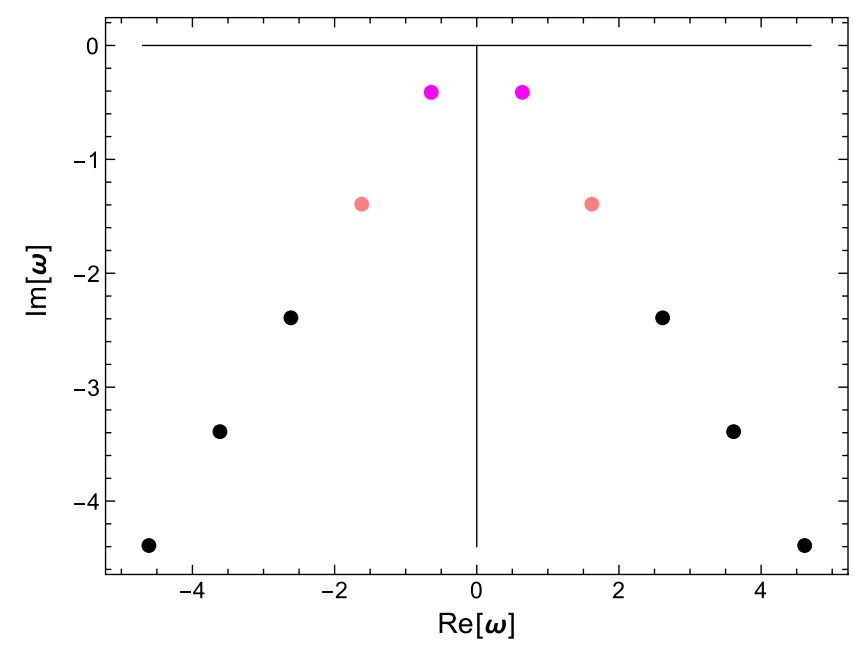

FIG. 6. Non-hydrodynamic QNMs of $\mathcal{N}=2^{*}$ black brane in the limit $\frac{m^{2}}{T^{2}} \rightarrow 0$ at $\mathfrak{q}=0$.

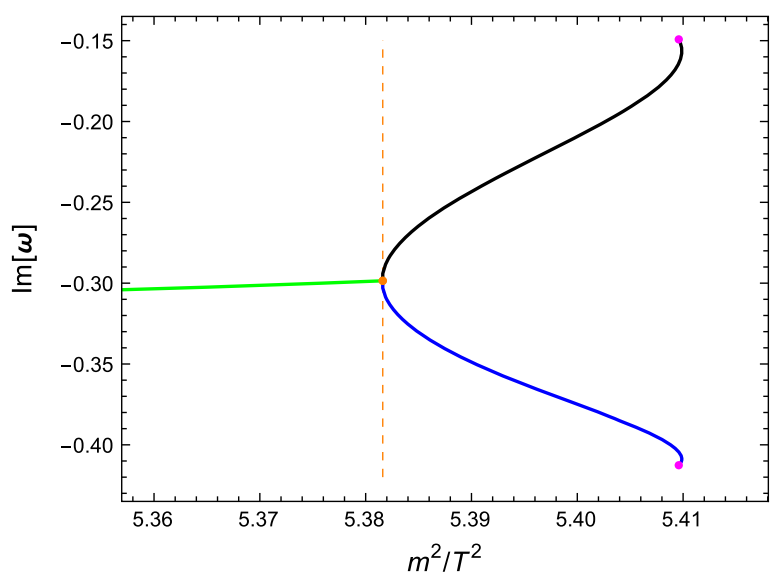

$$
0 \leq \frac{m^{2}}{T^{2}} \leq \frac{m^{2}}{T_{p}^{2}}
$$

While these QNMs start having both the real and the imaginary part of the frequency $\mathfrak{w}$ at $m=0$, they become purely dissipative for $T<T_{o}$,

$$
\frac{m^{2}}{T_{o}^{2}}=5.38162<\frac{m^{2}}{T_{p}^{2}},
$$

represented by a vertical dashed orange line. Note that these modes are stable in the thermodynamically unstable $\mathcal{N}=2^{*}$ black brane state of interest. In Fig. 8 we follow in the same fashion the pink QNMs of Fig. 6: they remain stable, and have $\operatorname{Re}[\mathfrak{w}] \neq 0$ up to the temperature (2.14).

\section{QNMs of the model at $K \neq 0$}

We fix the temperature at (2.14), i.e., in the thermodynamically unstable state of the (originally) $\mathcal{N}=2^{*}$ black brane, and study the QNMs as the horizon curvature $K$ becomes nonzero.

In Fig. 9 we present the dispersion of the $\ell=0$ (solid curves) and $\ell=1$ (dashed curves) modes of the $\mathcal{N}=2^{*}$ black hole with $\frac{K}{m^{2}}$, that originate from the magenta QNMs at $K=0$, see Fig. 7. These modes have $\operatorname{Re}[\mathfrak{w}]=0$. Initially stable $\ell=0$ mode becomes unstable (solid black curve) when

$$
\frac{K}{m^{2}}>\frac{K_{\ell=0}^{\text {stable }}}{m^{2}}=0.18290(4),
$$

represented by the vertical cyan line. The instability persists until

$$
\frac{K}{m^{2}}<\frac{K_{\ell=0}^{\text {unstable }}}{m^{2}}=0.62454(7),
$$

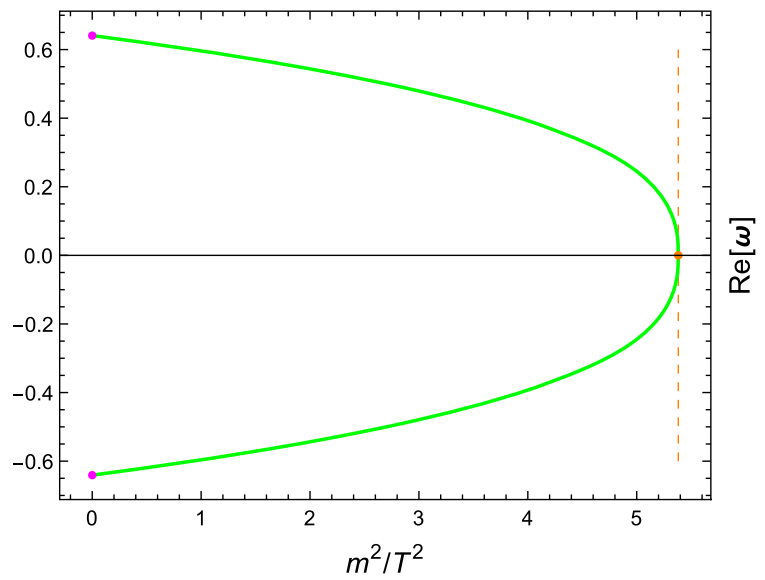

FIG. 7. We follow the pair of magenta QNMs of Fig. 6, at $\mathfrak{q}=0$, increasing $m$ to reach the thermodynamically unstable $\mathcal{N}=2^{*}$ black brane state (2.14). For $T_{p} \leq T<T_{o}$ these modes are purely dissipative. The temperature $T_{o}$ is represented by vertical dashed orange lines. 

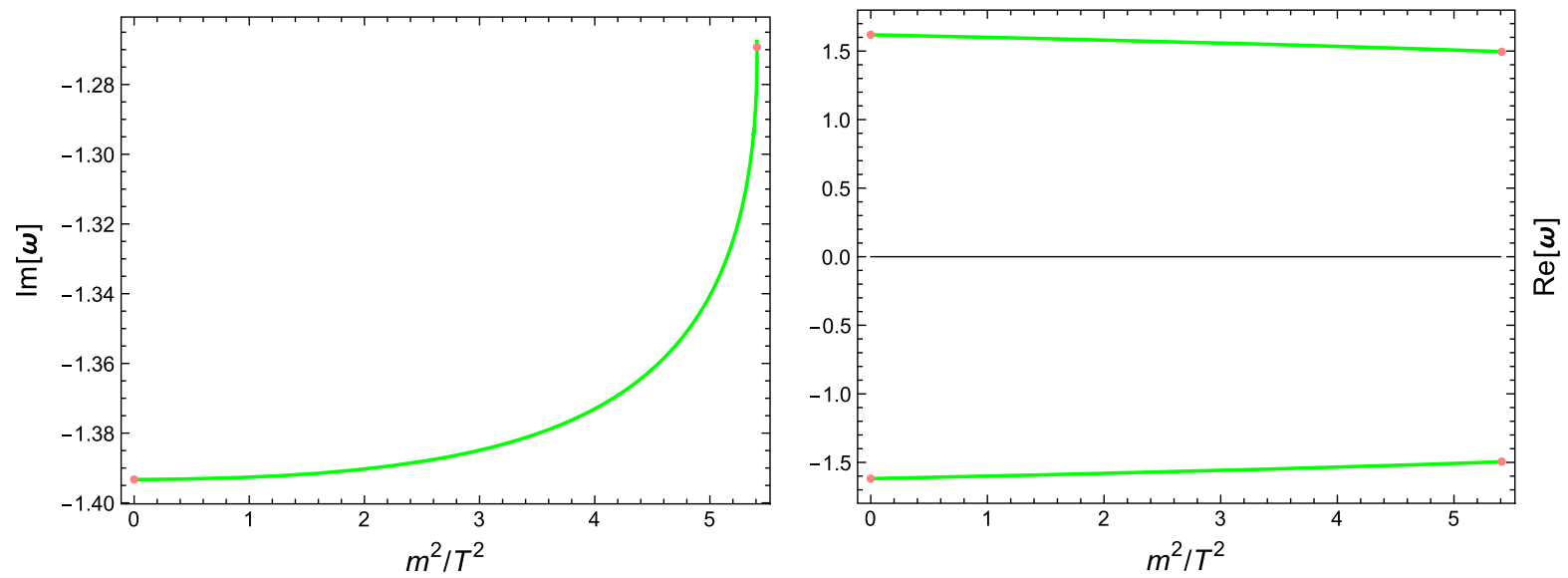

FIG. 8. We follow the pair of pink QNMs of Fig. 6, at $\mathfrak{q}=0$, increasing $m$ to reach the thermodynamically unstable $\mathcal{N}=2^{*}$ black brane state (2.14). These modes remain stable, with $\operatorname{Re}[\mathfrak{w}] \neq 0$.

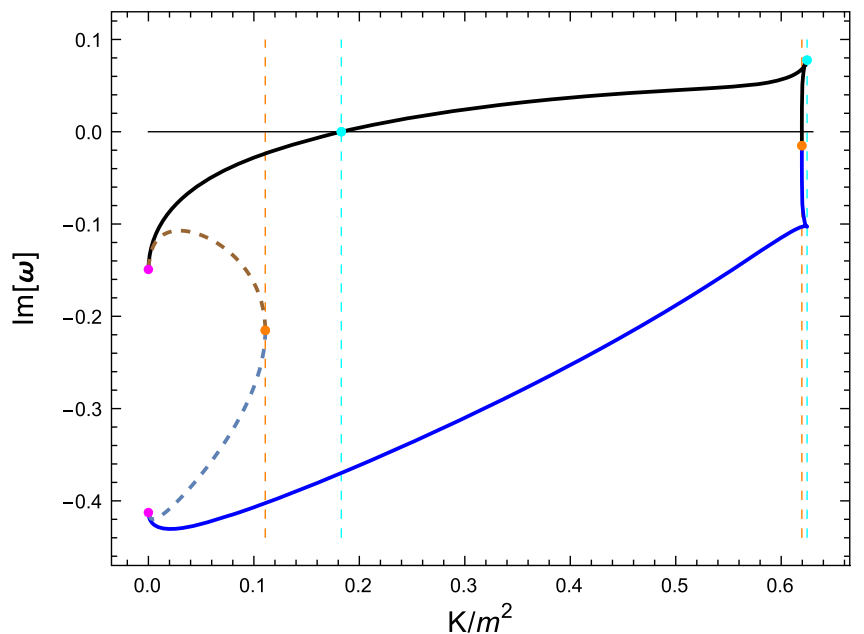

FIG. 9. $\ell=0$ (solid curves) and $\ell=1$ (dashed curves) QNMs of $\mathcal{N}=2^{*}$ black holes at fixed temperature (2.14) as functions of the curvature $K$. The $\ell=0$ (solid black) subbranch is unstable in the range of $K$ between the vertical dashed cyan lines. QNMs at $K=0$ originate from the (different) magenta modes of Fig. 7they coalesce as stable QNMs, represented by the orange dots.

represented by the cyan vertical dashed line. The QNM subbranches originating from different QNMs at $K=0$ (the magenta dots) coalesce at

$$
\begin{aligned}
& \ell=1: \frac{K_{o, 1}}{m^{2}}=0.11079(4), \\
& \ell=0: \frac{K_{o, 0}}{m^{2}}=0.61958(2),
\end{aligned}
$$

as the stable QNMs, represented by the orange dots. Since $K_{o, 0}<K_{\ell=0}^{\text {unstable }}$, this implies that close to $K_{\ell=0}^{\text {unstable }}$ the QNM subbranches can not be single-valued functions of $\frac{K}{m^{2}}$. As Fig. 10 shows this is indeed the case. See Sec. III for details how we are tracing the QNM curves.
In Fig. 11 we present the dispersion of the $\ell=0$ (solid green curves) and $\ell=1$ (dashed purple curves) modes of the $\mathcal{N}=2^{*}$ black hole with $\frac{K}{m^{2}}$, that originate from the pink QNMs at $K=0$, see Fig. 8 . These modes have $\operatorname{Re}[\mathfrak{w}] \neq 0$, and are stable. We follow them to $K>K_{\ell=0}^{\text {unstable }}$ and observe that their $\operatorname{Im}[\mathfrak{w}]$ start decreasing again. Close to $K_{\ell=0}^{\text {unstable }}$ these QNM subbranches are not single-valued functions of $\frac{K}{m^{2}}$ as well, see Fig. 12 .

In Fig. 13 we present the dispersion of the $\ell=2$ modes of the $\mathcal{N}=2^{*}$ black holes with $\frac{K}{m^{2}}$, that originate from the magenta QNMs at $K=0$ of Fig. 7 (grey and blue solid curves), and from the hydrodynamic QNM at $K=0$, represented with the cyan dot at $\mathfrak{w}=0$ (black and brown solid curves). The purely dissipative (stable) brown and grey branches (note that they originate from the hydrodynamic (the cyan dot) and the nonhydrodynamic (the magenta dot) modes at $K=0$ ) coalesce at

$$
\ell=2: \frac{K_{o, 2, b g}}{m^{2}}=0.0055428(2),
$$

represented by (the first) orange dot. A new (stable) subbranch, represented by the solid green lines starts of this orange dot. The QNM branch represented by the solid black curve contains the instabilities seen at $K=0$ in Fig. 5: these QNMs are unstable in the range between the two cyan dashed vertical lines

$$
0<\frac{K}{m^{2}}<\frac{K_{\ell=2}^{\text {unstable }}}{m^{2}}=0.0521(5)
$$

The $\operatorname{Re}[\mathfrak{w}]=0$ black and blue branches (note that they originate from the hydrodynamic (the cyan dot) and the nonhydrodynamic (the magenta dot) modes at $K=0$ ) coalesce at 

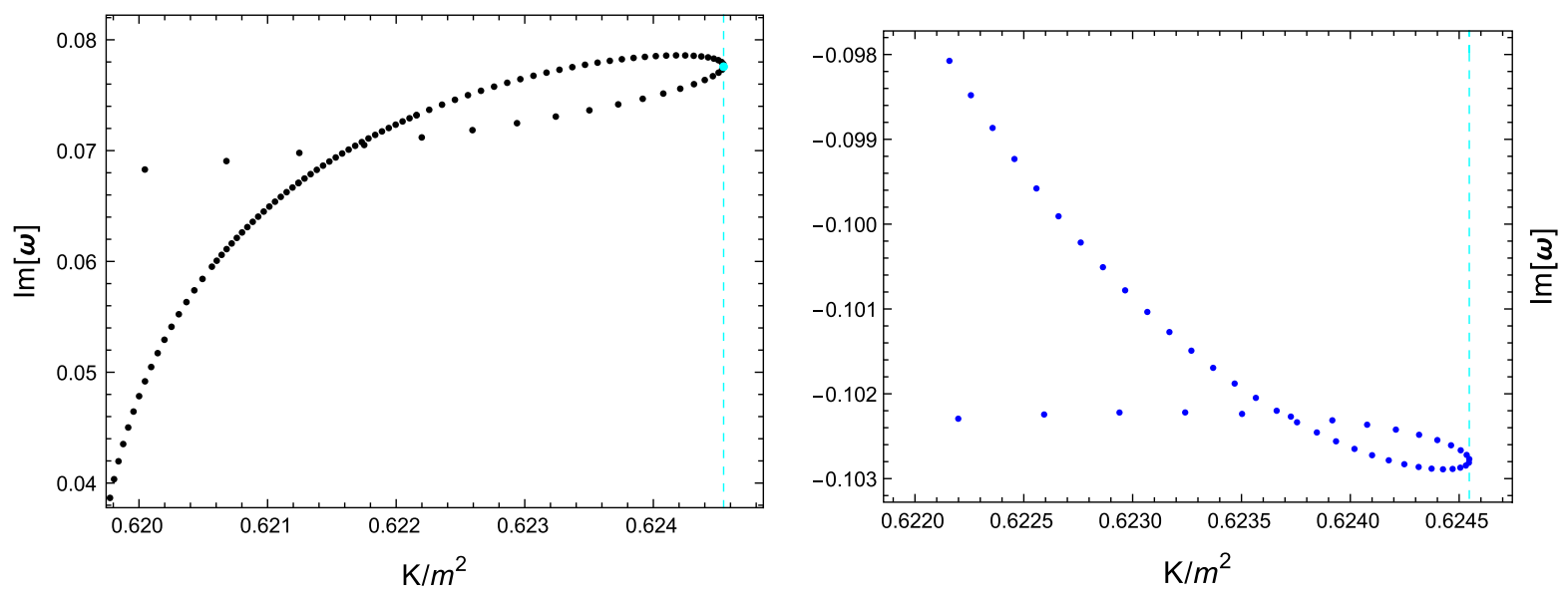

FIG. 10. $\ell=0 \mathcal{N}=2^{*}$ black hole QNMs, originated from the magenta modes of Fig. 7, are not single-valued functions of $\frac{K}{m^{2}}$ in the vicinity of $K_{\ell=0}^{\text {unstable }}$, represented by the vertical dashed cyan lines.
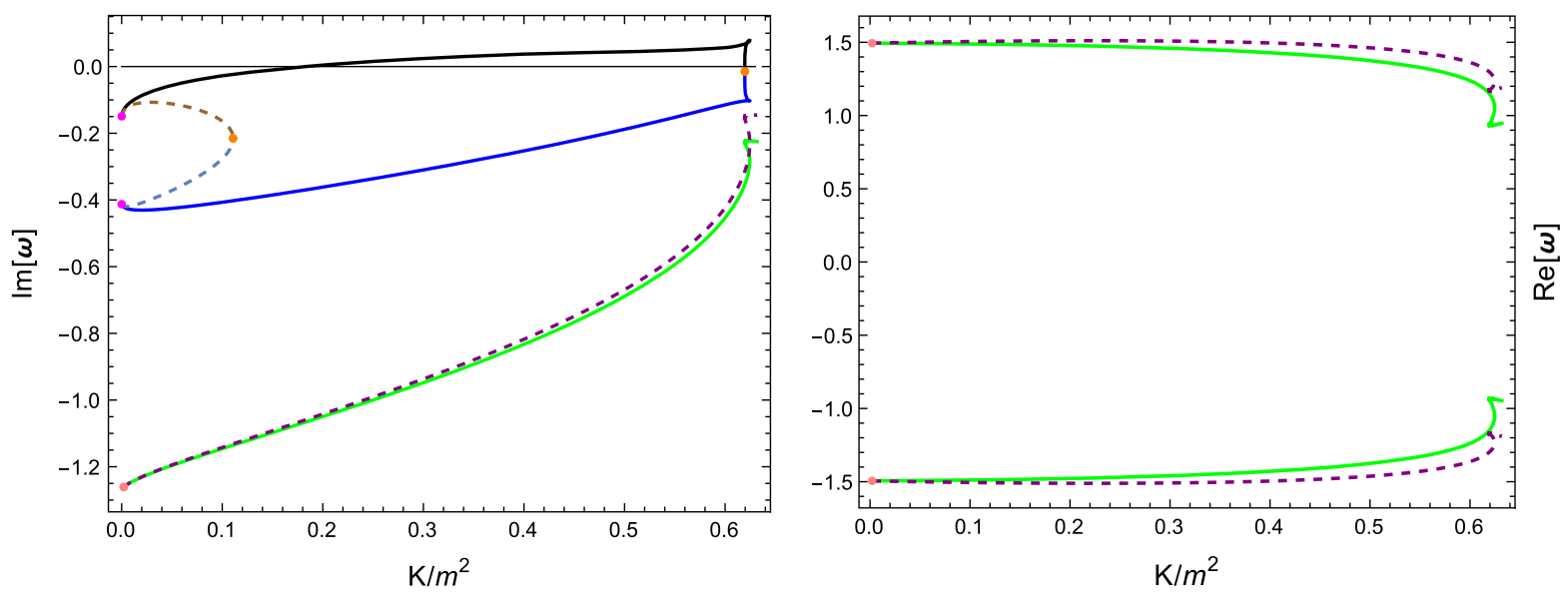

FIG. 11. Stable $\ell=0$ (solid green curves) and $\ell=1$ (dashed purple curves) QNMs of $\mathcal{N}=2^{*}$ black holes at fixed temperature (2.14) as functions of the curvature $K$, that originate from the pink QNMs at $K=0$, see Fig. 8.
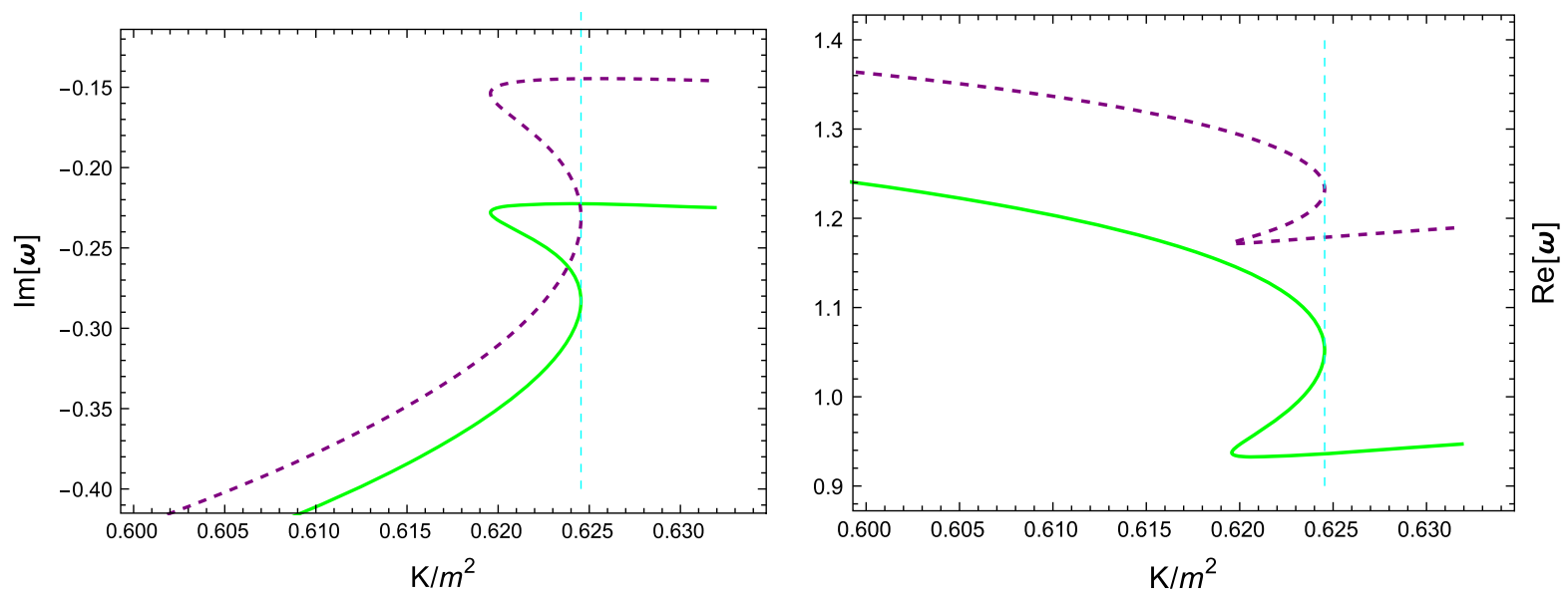

FIG. 12. Both $\ell=0$ (solid green curves) and $\ell=1$ (dashed purple curves) $\mathcal{N}=2^{*}$ black hole QNMs, originated from the pink modes of Fig. 8, are not single-valued functions of $\frac{K}{m^{2}}$ in the vicinity of $K_{\ell=0}^{\text {unstable }}$, represented by the vertical dashed cyan lines. 

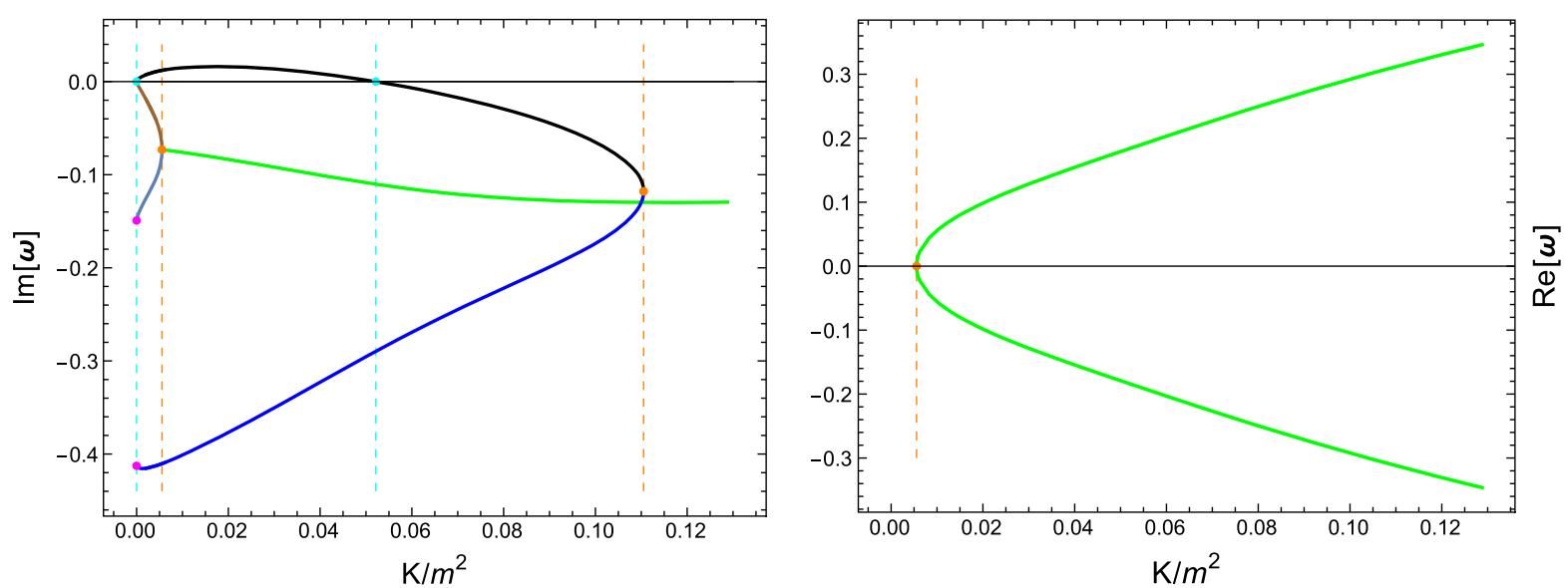

FIG. 13. $\ell=2$ QNMs of $\mathcal{N}=2^{*}$ black holes at fixed temperature (2.14) as functions of the curvature $K$. The solid black subbranch is unstable in the range of $K$ between the vertical dashed cyan lines. QNMs at $K=0$ originate from the magenta modes of Fig. 7 (grey and blue curves), and from the hydrodynamic QNM at $\mathfrak{w}=0$ (black and brown curves). The brown and grey subbranches coalesce, and continues as a green subbranch with $\operatorname{Re}[\mathfrak{w}] \neq 0$. No new subbranches originate once the black and the blue curves coalesce.
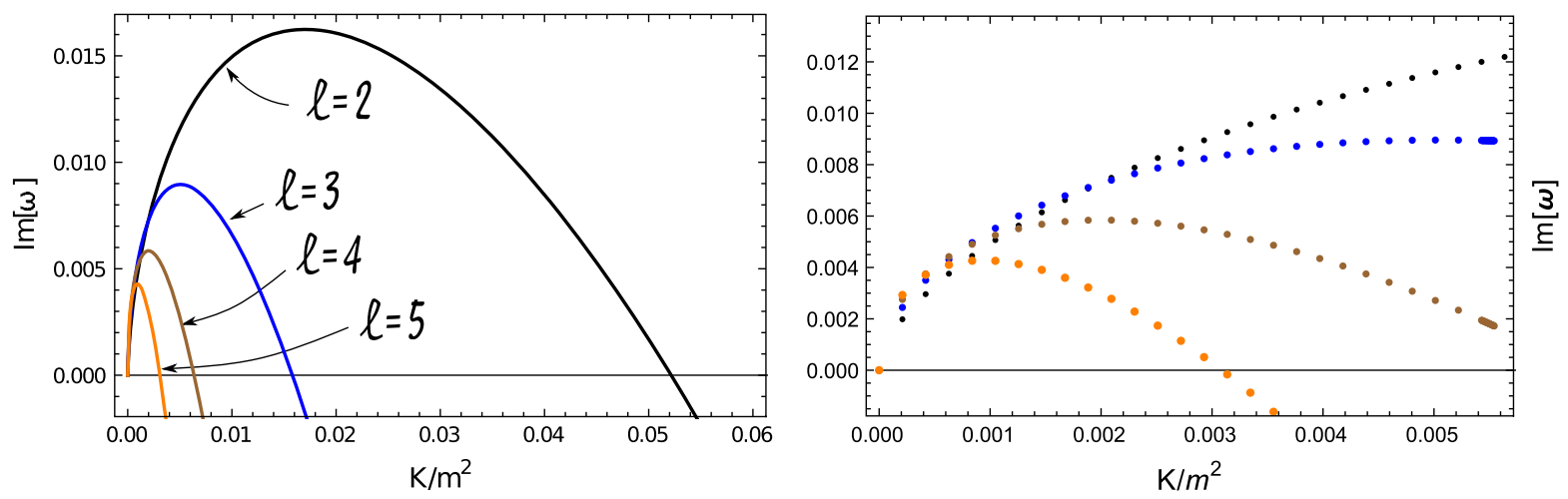

FIG. 14. Subbranches of the $\ell=2,3,4,5$ QNMs of $\mathcal{N}=2^{*}$ black holes at fixed temperature (2.14) that contain instabilities. Note that higher- $\ell$ QNMs are stabilized at successively smaller values of $\frac{K}{\mathrm{~m}^{2}}$.

$$
\ell=2: \frac{K_{o, 2, b b}}{m^{2}}=0.11050(5)
$$

represented by (the second) orange dot. No new subbranches start at the second orange dot. Interestingly, $K_{\ell=2}^{\text {unstable }}<K_{\ell=0}^{\text {stable }}$, as a result there is a finite range of $\frac{K}{m^{2}}$, see (2.7), where $\ell=2$ QNMs are already stable, but $\ell=0$ QNMs have yet to become unstable.

We conclude the summary of the $\mathcal{N}=2^{*}$ black hole QNMs at temperature (2.14) in Fig. 14: we show $\ell=$ $\{2,3,4,5\}$ subbranches that originate from the hydrodynamic $\mathfrak{w}=0$ QNM at $K=0$, and contain instabilities. All these modes are unstable as $\frac{K}{\mathrm{~m}^{2}} \rightarrow 0$, and higher- $\ell$ modes are stabilized at successively smaller values of $\frac{K}{\mathrm{~m}^{2}}$. Thus, the instabilities of $\ell>2$ modes do not further constrain the set of values of $\frac{K}{m^{2}}$ beyond that of the $\ell=2$ QNMs.

\section{TECHNICAL DETAILS}

The relevant effective action [19] describing $\mathcal{N}=2^{*}$ black holes is in the general class (A1) with $p=2$ :

$$
\begin{aligned}
\phi_{1} & \equiv \alpha, \quad \eta_{1}=12 ; \quad \phi_{2} \equiv \chi, \quad \eta_{2}=4, \\
V & =\frac{1}{4}\left(\frac{1}{3}\left(\frac{\partial W}{\partial \alpha}\right)^{2}+\left(\frac{\partial W}{\partial \chi}\right)^{2}\right)-\frac{4}{3} W^{2},
\end{aligned}
$$

where the superpotential $W$ is

$$
W=-e^{-2 \alpha}-\frac{1}{2} e^{4 \alpha} \cosh (2 \chi) .
$$

The scalars $\alpha$ and $\chi$ are the holographic dual to operators $\mathcal{O}_{2}$ and $\mathcal{O}_{3}$ correspondingly, describing the mass deformation of the maximally supersymmetric $\mathcal{N}=4$ Yang-Mills $^{6}$ $[20,22,37]$

\footnotetext{
${ }^{6}$ The field content of the $\mathcal{N}=4$ SYM theory includes the gauge field $A_{\mu}$, four Majorana fermions $\psi_{a}$ and three complex scalars $\phi_{i}$, where all of these fields are in the adjoint representation.
} 


$$
\begin{aligned}
\mathcal{L}_{\mathcal{N}=2^{*}}= & \mathcal{L}_{\mathcal{N}=4}-2\left[m_{b}^{2} \mathcal{O}_{2}+m_{f} \mathcal{O}_{3}\right], \\
\mathcal{O}_{2}= & \frac{1}{3} \operatorname{Tr}\left(\left|\phi_{1}\right|^{2}+\left|\phi_{2}\right|^{2}-2\left|\phi_{3}\right|^{2}\right), \\
\mathcal{O}_{3}= & -\operatorname{Tr}\left(i \psi_{1} \psi_{2}-\sqrt{2} g_{\mathrm{YM}} \phi_{3}\left[\phi_{1}, \phi_{1}^{\dagger}\right]\right. \\
& \left.+\sqrt{2} g_{\mathrm{YM}} \phi_{3}\left[\phi_{2}^{\dagger}, \phi_{2}\right]+\text { H.c. }\right) \\
& +\frac{2}{3} m_{f} \operatorname{Tr}\left(\left|\phi_{1}\right|^{2}+\left|\phi_{2}\right|^{2}+\left|\phi_{3}\right|^{2}\right) .
\end{aligned}
$$

The non-normalizable coefficients of the gravitational bulk scalars are the bosonic $m_{b}^{2}$ and the fermionic $m_{f}$ mass parameters. The thermodynamically unstable phase of the $\mathcal{N}=2^{*}$ plasma in $\mathbb{R}^{3}$ is present as long as [9]

$$
0 \leq \frac{m_{f}^{2}}{m_{b}^{2}}<1
$$

The effective action (3.2) can be consistently truncated to a single scalar $\alpha$, i.e., setting

$$
\chi \equiv 0 \Rightarrow m_{f}=0
$$

which we will do in the rest of this paper. To avoid cluttering we denote

$$
m_{b} \equiv m .
$$

\section{A. PW black holes and their thermodynamics}

We use the background geometry (A2) parameterization as in [38] and set

$$
c_{1}=\frac{f^{1 / 2}}{r h^{1 / 4}}, \quad c_{2}=\frac{1}{r h^{1 / 4}}, \quad c_{3}=\frac{h^{1 / 4}}{r f^{1 / 2}},
$$

where the radial coordinate

$$
r \in(0,+\infty)
$$

From (A5)-(A8) we obtain the second order equations

$$
\begin{gathered}
0=f^{\prime \prime}-\frac{3 f^{\prime}}{r}-\frac{5 h^{\prime} f^{\prime}}{4 h}+4 h K, \\
0=h^{\prime \prime}-\frac{5\left(h^{\prime}\right)^{2}}{4 h}-16 h\left(\alpha^{\prime}\right)^{2}, \\
0=\alpha^{\prime \prime}+a^{\prime}\left(\frac{f^{\prime}}{f}-\frac{3}{r}-\frac{5 h^{\prime}}{4 h}\right)+\frac{h^{1 / 2}}{6 r^{2} f}\left(e^{2 \alpha}-e^{-4 \alpha}\right),
\end{gathered}
$$

and the first order constraint

$$
\begin{aligned}
0= & \left(\alpha^{\prime}\right)^{2}+\frac{h K}{2 f}-\frac{\left(h^{\prime}\right)^{2}}{16 h^{2}}+\frac{h^{\prime} f^{\prime}}{16 f h}-\frac{h^{\prime}}{2 r h}+\frac{f^{\prime}}{4 r f} \\
& -\frac{1}{r^{2}}+\frac{h^{1 / 2}}{6 r^{2} f}\left(e^{2 \alpha}+\frac{1}{2} e^{-4 \alpha}\right) .
\end{aligned}
$$

Eqs. (3.9)-(3.12) are solved with the following asymptotics:

(i) in the UV, i.e., as $r \rightarrow 0$

$$
\begin{aligned}
f= & 1+16 r^{2} K-32 r^{3} \beta K+r^{4} f_{4,0}+\mathcal{O}\left(r^{5}\right), \quad(3.13) \\
h= & 16-64 \beta r+160 r^{2} \beta^{2}-320 r^{3} \beta^{3} \\
& +r^{4}\left(-\frac{128}{9} a_{2,0} a_{2,1}+\frac{416}{27} a_{2,1}^{2}+\frac{256}{3} a_{2,0}^{2}+560 \beta^{4}\right. \\
& \left.+\left(\frac{512}{3} a_{2,0} a_{2,1}-\frac{128}{9} a_{2,1}^{2}\right) \ln r+\frac{256}{3} a_{2,1}^{2} \ln ^{2} r\right) \\
& +\mathcal{O}\left(r^{5} \ln ^{2} r\right), \\
\alpha= & r^{2}\left(a_{2,0}+a_{2,1} \ln r\right)-\beta\left(2 a_{2,0}+a_{2,1}+2 a_{2,1} \ln r\right) r^{3} \\
& +r^{4}\left(3 a_{2,0} \beta^{2}+\frac{5}{2} a_{2,1} \beta^{2}-8 K a_{2,1}+a_{2,0}^{2}-2 a_{2,0} a_{2,1}\right. \\
& +\frac{3}{2} a_{2,1}^{2}+\left(3 a_{2,1} \beta^{2}+2 a_{2,0} a_{2,1}-2 a_{2,1}^{2}\right) \ln r \\
& \left.+a_{2,1}^{2} \ln ^{2} r\right)+\mathcal{O}\left(r^{5} \ln ^{2} r\right) ;
\end{aligned}
$$

(ii) in the IR, i.e., as $y \equiv \frac{1}{r} \rightarrow 0$

$$
\begin{aligned}
f= & f_{1}^{h} y-\left(\frac{5}{3} r_{0}^{2}\left(h_{0}^{h}\right)^{1 / 2}+7 h_{0}^{h} K+\frac{5\left(h_{0}^{h}\right)^{1 / 2}}{6 r_{0}^{4}}\right) y^{2} \\
& +\mathcal{O}\left(y^{3}\right), \\
\hat{h}= & h_{0}^{h}-\left(\frac{8\left(h_{0}^{h}\right)^{3 / 2} r_{0}^{2}}{3 f_{1}^{h}}+\frac{8\left(h_{0}^{h}\right)^{2} K}{f_{1}^{h}}+\frac{4\left(h_{0}^{h}\right)^{3 / 2}}{3 f_{1}^{h} r_{0}^{4}}\right) y \\
& +\mathcal{O}\left(y^{2}\right), \\
\alpha= & \ln r_{0}-\frac{\left(r_{0}^{6}-1\right)\left(h_{0}^{h}\right)^{1 / 2}}{6 f_{1}^{h} r_{0}^{4}} y+\mathcal{O}\left(y^{2}\right),
\end{aligned}
$$

where we defined

$$
\hat{h} \equiv y^{-4} h
$$

The non-normalizable coefficient $a_{2,1}$ of the bulk scalar $\alpha$ is related to the mass parameter (3.6) as follows [20,22]

$$
m^{2}=\frac{3}{8} a_{2,1} .
$$

$\beta$ is a residual gauge parameter of the background geometry (3.7) parameterization associated with the constant $\lambda$ rescaling of radial coordinate (3.8), $r \rightarrow r \lambda$ or $y \rightarrow \frac{y}{\lambda}$ : 


$$
\begin{array}{rlrl}
\beta & \rightarrow \beta \lambda, \quad K \rightarrow K \lambda^{2}, \\
a_{2,1} & \rightarrow a_{2,1} \lambda^{2}, & a_{2,0} \rightarrow\left(a_{2,0}+a_{2,1} \ln \lambda\right) \lambda^{2}, \\
f_{4,0} & \rightarrow f_{4,0} \lambda^{2}, & f_{1}^{h} \rightarrow \frac{f_{1}^{h}}{\lambda}, \\
h_{0}^{h} & \rightarrow \frac{h_{0}^{h}}{\lambda^{4}}, & r_{0} \rightarrow r_{0} .
\end{array}
$$

Because (3.19) acts on all dimensionful parameters ( $K$ and $m$ specifically), the results of the holographic renormalization of the model has to be expressed as dimensionless quantities (2.9). The holographic renormalization of the $\mathcal{N}=2^{*}$ model has been discussed extensively in the past, so we present the results only $[26,39,40]$ :

$$
\begin{aligned}
\hat{\mathcal{E}}= & \frac{1}{24 \pi^{4}}\left(-\frac{2}{3}-\frac{2 f_{4,0}}{3 a_{2,1}^{2}}+\frac{128 K^{2}}{3 a_{2,1}^{2}}-\frac{8}{3} \ln 2\right. \\
& \left.-\frac{4}{3} \ln a_{2,1}+\frac{8 a_{2,0}}{3 a_{2,1}}+\frac{32 \beta^{2} K}{a_{2,1}^{2}}\right), \\
\hat{s}= & \frac{2^{7 / 2}}{3^{5 / 2} \pi^{3}\left(h_{0}^{h}\right)^{3 / 4} a_{2,1}^{3 / 2}}, \\
\frac{T}{m}= & \frac{f_{1}^{h}}{6^{1 / 2} \pi\left(h_{0}^{h}\right)^{1 / 2} a_{2,1}^{1 / 2}} .
\end{aligned}
$$

Note that (3.20) are left invariant under (3.19). The basic thermodynamic relation,

$$
\mathcal{F}=\mathcal{E}-s T
$$

is automatically enforced by the holographic renormalization [39], while the first law of thermodynamics,

$$
d \hat{\mathcal{E}}=\left.\frac{T}{m} d \hat{s}\right|_{\frac{K}{m^{2}}=\text { const }},
$$

must be verified numerically. We always check (3.22) in numerical constructions of the $\mathcal{N}=2^{*}$ black brane/black hole geometries-a sample of tests, for $\frac{K}{m^{2}}=0$ and $\frac{K}{m^{2}}=1$, is shown in Fig. 16.

\section{B. Helicity $h=0$ QNMs of the PW black holes}

We suppress the $h=0$ superscript, and refer to a single scalar index as $s=0$, rather then $s=(0, j)$. Using the background parametrization (3.7), we obtain from (A12):

$$
\begin{aligned}
& 0=\mathcal{D}_{2} F_{2}-W_{2,2} F_{2}-W_{2,0} F_{0}, \\
& 0=\mathcal{D}_{2} F_{0}-W_{2,0} F_{2}-W_{0,0} F_{0},
\end{aligned}
$$

where the second-order differential operator $\mathcal{D}_{2}$ [coming from $\square$ on the background geometry (A2)] is

$$
\begin{aligned}
\mathcal{D}_{2} F(t, r) \equiv & -\frac{h^{1 / 2} r^{2}}{f} \partial_{t t}^{2} F+\frac{r^{2} f}{h^{1 / 2}} \partial_{r r}^{2} F \\
& +\left(\frac{r^{2} f^{\prime}}{h^{1 / 2}}-\frac{5 r^{2} f h^{\prime}}{4 h^{3 / 2}}-\frac{3 r f}{h^{1 / 2}}\right) \partial_{r} F \\
& -h^{1 / 2} r^{2} k^{2} F
\end{aligned}
$$

and

$$
\begin{aligned}
W_{2,2}=- & \frac{1024 r^{4} h^{7 / 2} f k^{2}\left(3 K-k^{2}\right)\left(\alpha^{\prime}\right)^{2}}{G^{2}}+\frac{32 r^{2} h^{3 / 2} f\left(h^{\prime} r+4 h\right)^{2}\left(k^{2}-3 K\right)^{2}}{G^{2}} \\
- & \frac{2 r\left(3 K-k^{2}\right)}{3 G h^{1 / 2}}\left(16 h^{3} k 2 r^{2}+6 h h^{\prime} f^{\prime} r^{2}-9 f\left(h^{\prime}\right)^{2} r^{2}+24 h^{2} f^{\prime} r-72 h f h^{\prime} r-144 h^{2} f\right) \\
- & \frac{4}{3} k^{2} h^{1 / 2} r^{2}, \\
W_{2,0}= & \frac{8 \sqrt{2 k^{2}\left(k^{2}-3 K\right)}}{3 G^{2}}\left(9 h^{1 / 2} f r^{2}\left(h^{\prime} r+4 h\right)\left(16 K h^{2} r-8 h^{2} k^{2} r+4 h f^{\prime}+h^{\prime} f^{\prime} r\right) \alpha^{\prime}\right. \\
& \left.+2 h^{2} r\left(8 h^{2} k^{2} r-12 h f^{\prime}-3 h^{\prime} f^{\prime} r\right)\left(e^{2 \alpha}-e^{-4 \alpha}\right)-576 h^{5 / 2} f f^{\prime} r^{4}\left(\alpha^{\prime}\right)^{3}\right), \\
W_{0,0}= & -\frac{1}{3 h G^{2}}\left(96 h^{2} f^{\prime} r\left(e^{2 \alpha}-e^{-4 \alpha}\right)\left(8 h^{2} k^{2} r-12 h f^{\prime}-3 h^{\prime} f^{\prime} r\right) \alpha^{\prime}+288 h^{1 / 2} r^{2}\left(\alpha^{\prime}\right)^{2}\right. \\
& \times\left(32 K h^{4} f k^{2} r^{2}-16 h^{4} f k^{4} r^{2}+8 h^{3}\left(f^{\prime}\right)^{2} k^{2} r^{2}-24 h^{3} f f^{\prime} k^{2} r-6 h^{2} f h^{\prime} f^{\prime} k^{2} r^{2}\right. \\
& \left.-12 h^{2}\left(f^{\prime}\right)^{3} r-3 h h^{\prime}\left(f^{\prime}\right)^{3} r^{2}+48 h^{2} f\left(f^{\prime}\right)^{2}+24 h f h^{\prime}\left(f^{\prime}\right)^{2} r+3 f\left(h^{\prime}\right)^{2}\left(f^{\prime}\right)^{2} r^{2}\right) \\
& \left.-13824 h^{5 / 2} f\left(f^{\prime}\right)^{2} r^{4}\left(\alpha^{\prime}\right)^{4}\right)-\frac{1}{3}\left(e^{2 \alpha}+2 e^{-4 \alpha}\right),
\end{aligned}
$$


with $k$ given by (A11) and

$$
G \equiv 8 h^{2} k^{2} r-12 h f^{\prime}-3 h^{\prime} f^{\prime} r
$$

Generically, $F_{0}$ and $F_{2}$, as well as $\mathfrak{w}=\mathfrak{w}(\mathfrak{q})$, are complex. We need to impose the normalizable boundary conditions as $r \rightarrow 0$, and the incoming wave boundary conditions at the black brane/black hole horizon, i.e., as $y \equiv \frac{1}{r} \rightarrow 0$. We can explicitly factor the boundary conditions, and the harmonic time dependence, redefining $F_{0}$ and $F_{2}$ as

$$
\begin{aligned}
& F_{0}(t, r)=(1+r)^{i \mathfrak{w} / 2} \frac{r^{2}}{1+r^{2}} e^{-i 2 \pi T \mathfrak{w} t} f_{0}(r), \\
& F_{2}(t, r)=(1+r)^{i \mathfrak{w} / 2} \frac{r^{2}}{1+r^{2}} e^{-i 2 \pi T \mathfrak{w} t} f_{2}(r),
\end{aligned}
$$

which renders both $f_{0}(r)$ and $f_{2}(r)$ regular and $\mathcal{O}(1)$ both at the boundary and at the horizon. While the boundary normalization of the master scalar $F_{2} \sim \mathcal{O}\left(r^{2}\right)$ near the $\mathrm{AdS}_{5}$ boundary is independent of any presence of the bulk gravitational scalars, the boundary normalization of the master scalar $F_{0} \sim \mathcal{O}\left(r^{2}\right)$ reflects the fact that this scalar is associated with the fluctuations of the gravitational bulk scalar $\alpha$ dual to an operator of the conformal dimension $\Delta=2$. The incoming wave boundary condition at the horizon, i.e., $F \propto y^{-i \mathfrak{w} / 2}$ is universal for all the master scalars. Further introducing

$$
\begin{aligned}
f_{0} & =f_{0, \operatorname{Re}}+i f_{0, \operatorname{Im}}, \quad f_{2}=f_{2, \operatorname{Re}}+i f_{2, \operatorname{Im}} \\
\mathfrak{w} & =\mathfrak{w}_{\operatorname{Re}}+i \mathfrak{w}_{\mathrm{Im}},
\end{aligned}
$$

we obtain from (3.23) four (generically coupled) second order linear ODEs for

$$
\left\{f_{0, \operatorname{Re}}, f_{0, \mathrm{Im}}, f_{2, \operatorname{Re}}, f_{2, \mathrm{Im}}\right\} .
$$

Because of the linearity, there is an arbitrary complex overall normalization of solution; we fix this normalization imposing

$$
\begin{aligned}
& \ell \geq 2: \lim _{r \rightarrow 0} f_{2, \operatorname{Re}}=1, \quad \lim _{r \rightarrow 0} f_{2, \operatorname{Im}}=0, \\
& \ell<2: \lim _{r \rightarrow 0} f_{0, \mathrm{Re}}=1, \quad \lim _{r \rightarrow 0} f_{0, \mathrm{Im}}=0 .
\end{aligned}
$$

The QNM equations for (3.31) are solve with the following asymptotics: (i) in the UV, i.e., as $r \rightarrow 0$ (without loss of generality, we work in the gauge $\beta=1$-see (3.13)-(3.15))

$$
\begin{aligned}
f_{2, \operatorname{Re}}= & 1+\left(-2+\frac{1}{2} \mathfrak{w}_{\mathrm{Im}}\right) r+\mathcal{O}\left(r^{2}\right), \\
f_{2, \mathrm{Im}}= & -\frac{1}{2} \mathfrak{w}_{\mathrm{Re}} r+\mathcal{O}\left(r^{2}\right), \\
f_{0, \operatorname{Re}}= & f_{0, \operatorname{Re}, 0} \\
& +\left(-2 f_{0, \operatorname{Re}, 0}+\frac{1}{2} f_{0, \operatorname{Im}, 0} \mathfrak{w}_{\mathrm{Re}}+\frac{1}{2} f_{0, \operatorname{Re}, 0} \mathfrak{w}_{\mathrm{Im}}\right) r \\
& +\mathcal{O}\left(r^{2}\right), \\
f_{0, \mathrm{Im}}= & f_{0, \operatorname{Im}, 0} \\
& +\left(-2 f_{0, \operatorname{Im}, 0}+\frac{1}{2} f_{0, \operatorname{Im}, 0} \mathfrak{w}_{\mathrm{Im}}-\frac{1}{2} f_{0, \operatorname{Re}, 0} \mathfrak{w}_{\mathrm{Re}}\right) r \\
& +\mathcal{O}\left(r^{2}\right) ;
\end{aligned}
$$

(ii) in the IR, i.e., as $y \equiv \frac{1}{r} \rightarrow 0$

$$
\begin{array}{ll}
f_{2, \operatorname{Re}}=f_{2, \operatorname{Re}, 0}^{h}+\mathcal{O}(y), & f_{2, \operatorname{Im}}=f_{2, \operatorname{Im}, 0}^{h}+\mathcal{O}(y), \\
f_{0, \operatorname{Re}}=f_{0, \operatorname{Re}, 0}^{h}+\mathcal{O}(y), & f_{0, \operatorname{Im}}=f_{0, \operatorname{Im}, 0}^{h}+\mathcal{O}(y) .
\end{array}
$$

Note that, for a fixed background and $\mathfrak{q}$, the solution is characterized in total by 8 parameters

$$
\begin{gathered}
\left\{\mathfrak{w}_{\mathrm{Re}}, \mathfrak{w}_{\mathrm{Im}}, f_{0, \operatorname{Re}, 0}, f_{0, \operatorname{Im}, 0}, f_{2, \operatorname{Re}, 0}^{h},\right. \\
\left.f_{2, \operatorname{Im}, 0}^{h}, f_{0, \operatorname{Re}, 0}^{h}, f_{0, \operatorname{Im}, 0}^{h}\right\},
\end{gathered}
$$

precisely as needed to specify a solution of 4 second order ODEs.

We now highlight the reduction of the set of the QNM equations in some special cases.

(i) For branches of the QNM modes with $\operatorname{Re}[\mathfrak{w}]=0$, as in Figs. 5, 13, and 14 we can consistently set

$$
f_{2, \operatorname{Im}}(r) \equiv 0, \quad f_{0, \operatorname{Im}}(r) \equiv 0, \quad \mathfrak{w}_{\mathrm{Re}}=0,
$$

correspondingly eliminating, in addition to $\mathfrak{w}_{\mathrm{Re}}$, $f_{0, \operatorname{Im}, 0}, f_{2, \operatorname{Im}, 0}^{h}$, and $f_{0, \operatorname{Im}, 0}^{h}$ from the parameter list (3.35) — we are left with 2 second order differential equations and 4 specifying parameters.

(ii) Special case harmonics $\ell=0$ and $\ell=1$ can be treated by dropping the master equation for $f_{2}$, and setting $k=0$ or $k=\sqrt{3 K}$ (for $\ell=0$ and $\ell=1$ correspondingly) in the remaining equations for $f_{0, \operatorname{Re}}$ and $f_{0, \mathrm{Im}}$. Note that in this case we need to normalize the solution as in the second line in (3.32). QNM branches of this type appear in Figs. 7 and 8 . 
(iii) Yet further reduction occurs for $\ell=0$ and $\ell=1$ if the QNMs have $\operatorname{Re}[\mathfrak{w}]=0$, as in Figs. 7 and 9. Here we have a single second order equation for the function $f_{0, \operatorname{Re}}$ (with $k=0$ or $k=\sqrt{3 K}$ ) and a pair of specifying parameters $\mathfrak{w}_{\mathrm{Im}}$ and $f_{0, \mathrm{Re}, 0}^{h}$.

(iv) We included $K=0$ (the black brane) case of QNMs at $k=0$ with $\ell=0$ case covered above.

We add some practical remarks for the QNM computation.

(i) In solving the QNM boundary value problems we use the shooting method developed in [41].

(ii) To obtain results for the QNMs reported in Figs. 7 and 8 we set $K=0$, and produce the data sets of backgrounds changing in small increments parameter $a_{2,1}$ in (3.13)-(3.15) from $a_{2,1}=0$ (the pure $\mathrm{AdS}_{5}$-Schwarzschild black brane) to the value $a_{2,1}=0.09158879$. We fix the gauge parameter $\beta=1$. This allows a black brane construction covering the range of $\frac{m^{2}}{T^{2}}$ reported in Figs. 1 and 4 . Note that a monotonic change in $a_{2,1}$ translates into nonmonotonic dependence of $\frac{m^{2}}{T^{2}}$, allowing to cover both the thermodynamically stable and the unstable phases. The thermodynamically unstable state of interest (2.14) is within the scanned range. For each black brane background we compute the QNMs, using as initial seeds the $\mathrm{AdS}_{5}$-Schwarzschild black brane QNMs reported in Fig. 6.

(iii) To obtain results for the QNMs reported in Figs. $9-13$ we produce $\mathcal{N}=2^{*}$ black hole backgrounds keeping mass-to-temperature ratio $\frac{m^{2}}{T^{2}}$ fixed at (2.14), and changing $K$ from zero in small increments. For each generated black hole background we compute the QNMs, using as initial seeds the QNMs of the $\mathcal{N}=2^{*}$ black brane. Since we work in a fixed $\beta=1$ gauge, the physical curvature is not $K$, but rather its dimensionless analog, $\frac{K}{m^{2}}$.
Similar to the black brane constructions from variation of $a_{2,1}$, we observe that while we monotonically increase $K$ at fixed $\frac{m^{2}}{T^{2}}, \frac{K}{m^{2}}$ varies nonmonotonicallythis is the origin of the nonsingle valuedness of the QNM spectra in Figs. 10 and 12.

\section{Helicity $h=0$ QNMs of PW black branes in the limit $a_{2,1} \rightarrow 0$}

The purpose of this section is to discuss the limit of the general QNM equations (3.23) when $K=0$ and $a_{2,1}=0$. This is a pure $\mathrm{AdS}_{5}$-Schwarzschild black brane limit, and could be helpful to the reader to study prior to tackling the general case covered in Sec. III B.

Solving (3.9)-(3.12) with $K=0, a_{2,1}=0$ and the boundary conditions (3.13)-(3.16) we find:

$$
\begin{aligned}
& \alpha \equiv 0, \quad h=\frac{16}{(\beta r+1)^{4}}, \\
& f=\frac{(2 \beta r+1)\left(2 \beta^{2} r^{2}+2 \beta r+1\right)}{(\beta r+1)^{4}} .
\end{aligned}
$$

As in Sec. III B, we set the gauge parameter $\beta=1$.

Given (3.37), the general QNM equations (3.23) decouple:

$$
\begin{aligned}
0= & \partial_{t t}^{2} F_{0}-\frac{(2 r+1)^{2}\left(2 r^{2}+2 r+1\right)^{2}}{16(r+1)^{4}} \partial_{r r}^{2} F_{0} \\
& -\frac{(2 r+1)\left(2 r^{2}+2 r+1\right)\left(4 r^{4}-10 r^{2}-10 r-3\right)}{16 r(r+1)^{5}} \partial_{r} F_{0} \\
& +\frac{(2 r+1)\left(2 r^{2}+2 r+1\right)\left(4 k^{2} r^{2}-r^{2}-2 r-1\right)}{4(r+1)^{4} r^{2}} F_{0},
\end{aligned}
$$

(i) Eq. (3.38) has a smooth limit $k \rightarrow 0$. Redefining $F_{0}(t, r)$ as in (3.29) and solving the resulting equations at $k=0$, we produce the spectrum of $\Delta=2$ QNMs in $\mathrm{AdS}_{5}$-Schwarzschild black brane reported in Fig. 6, reproducing the appropriate results of [42].

(ii) Because of the factor $\left(8 k^{2} r^{2}+16 k^{2} r+3 r^{2}+8 k^{2}\right)$ in the denominator of the equation (3.39), the $k \rightarrow 0$ 

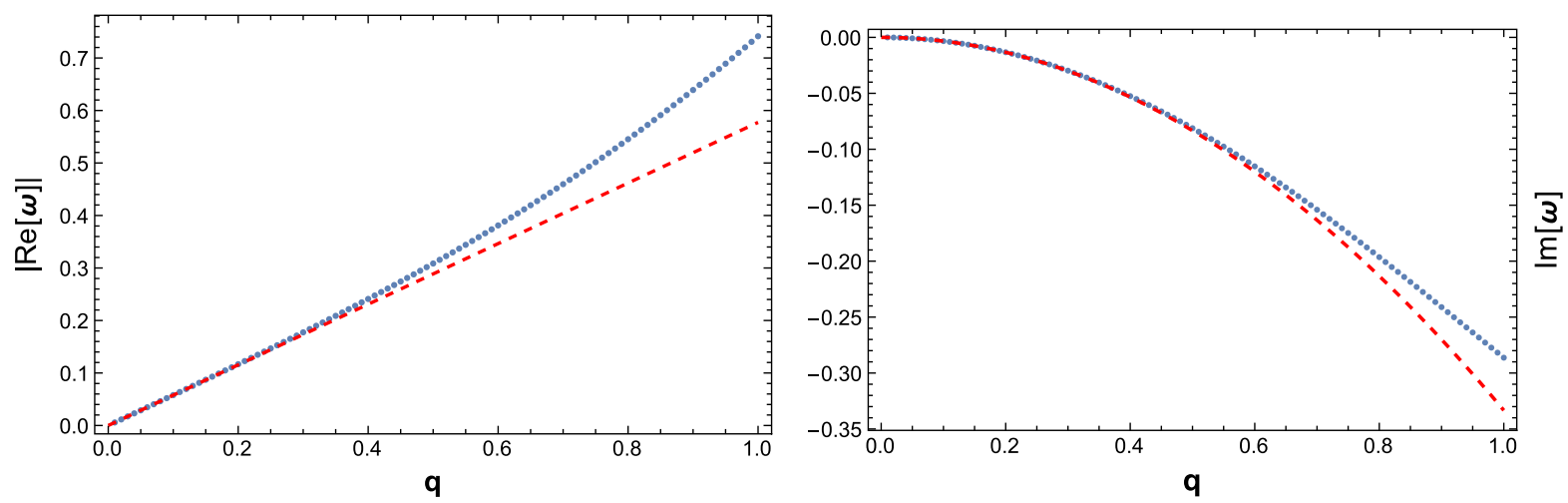

FIG. 15. The hydrodynamic QNM of $\mathrm{AdS}_{5}$-Schwarzschild black brane computed in the framework of Appendix A. Dashed red curves represent the leading $\mathfrak{q} \rightarrow 0$ approximation, see (3.40).

and $r \rightarrow 0$ limits do not commute. We can still solve, say for the hydrodynamic QNM, using the redefinition (3.29) and generate the spectrum $\mathfrak{w}(\mathfrak{q})$ for $\mathfrak{q} \geq \frac{1}{100}$. The results are presented in Fig. 15, along with the expected small $\mathfrak{q}$ holographic-CFT approximation (dashed red curves):

$$
\mathfrak{w}(\mathfrak{q})= \pm \frac{1}{\sqrt{3}} \mathfrak{q}-\frac{1}{3} \mathfrak{q}^{2}+\mathcal{O}\left(\mathfrak{q}^{3}\right)
$$

At $\mathfrak{q}=1$ we can compare our results with those reported in Appendix E of [18] (JRR):

this work: $\mathfrak{w}(1)= \pm 0.7414299655(2)$

$$
-0.2862800072(6) i
$$

JRR: $\mathfrak{w}(1)= \pm 0.7414299655(?)$

$$
-0.2862800072(?) i \text {, }
$$

where (?) denotes the unreported truncation in [18].

\section{CONCLUSIONS}

In this paper we extended the master field framework of black brane/black hole QNM computations to Einsteinmultiscalar gravitational effective actions of the type (A1).

As an application, we discussed the stabilization of the thermodynamically unstable $\mathcal{N}=2^{*}$ black brane horizons, as the boundary gauge theory is compactified on $S^{3}$ of a curvature scale $K$. The initial instability in the helicity $h=0$ hydrodynamic sector, present for small curvature, is cured once $K>K_{\ell=2}^{\text {unstable }}$. Surprisingly, we found that while $\ell=0$ QNMs are stable for $K \in\left[0, K_{\ell=2}^{\text {unstable }}\right]$, they develop an instability for yet larger $K>K_{\ell=0}^{\text {stable }}>K_{\ell=2}^{\text {unstable }}$. They can ultimately be stabilized for $K>K_{\ell=0}^{\text {unstable }} \cdot \ell=1$ QNMs in the helicity $h=0$ sector in the model discussed are always stable.

In the example of the $\mathcal{N}=2^{*}$ holography, the extended thermodynamically unstable horizons can be alternatively stabilized when the space of the boundary gauge theory is compactified on sufficiently small three-torus: the unstable hydrodynamic modes are simply projected from the spectrum. ${ }^{7}$ However, toroidal compactification would not be able to cure the instability of the holographic conformal order, present in the nonhydrodynamic sector at zero spatial momentum [15].

Many avenues are left open for future studies:

(i) it would be interesting to understand when Klebanov-Strassler black holes [38] become dynamically stable;

(ii) it would be interesting to explore whether the holographic conformal order [13-15] can be stabilized on $S^{d}$, before it is destroyed;

(iii) what aspects of the stabilization when black branes are deformed to black holes are universal? are $\ell=1$ harmonics in the helicity $h=0$ sector always stable?

Finally, in this work we considered effective actions relevant to holographic models without the bulk gauge field, correspondingly without conserved global charges of the thermal states of the boundary gauge theory. It is only in this setting that one straightforwardly relate via (1.3) the thermodynamic and the dynamical instabilities [8]. In the presence of a conserved $U(1)$ charge density $\rho$ and a chemical potential $\mu$ the story is more nuanced, ${ }^{8}$ as the expression for the speed of the sound waves (1.3) becomes more complicated in the charged plasma:

$c_{s}^{2}=\left((\mathcal{E}+P) \frac{\partial(P, \rho)}{\partial(T, \mu)}+\rho \frac{\partial(\mathcal{E}, P)}{\partial(T, \mu)}\right)\left((\mathcal{E}+P) \frac{\partial(\mathcal{E}, \rho)}{\partial(T, \mu)}\right)^{-1}$.

Additionally, in the presence of the global charges, one can no longer establish in full generality the stability of the QNMs in the helicity $h=1$ sector. It would be interesting to explore this in the future.

\footnotetext{
${ }^{7}$ We would like to thank David Mateos for pointing this out. ${ }^{8}$ See $[11]$.
} 


\section{ACKNOWLEDGMENTS}

This research is supported in part by Perimeter Institute for Theoretical Physics. Research at Perimeter Institute is supported in part by the Government of Canada through the Department of Innovation, Science and Economic Development Canada and by the Province of Ontario through the Ministry of Colleges and Universities. This work was further supported by NSERC through the Discovery Grants program.

\section{APPENDIX A: MASTER EQUATIONS OF EINSTEIN-SCALAR BLACK HOLES IN $D=5$}

In this section we extend the work of [18] to black holes in theories of Einstein gravity in $D=5$ space-time dimensions with multiple scalars. ${ }^{9}$ We adhere to the notations of [18] as much as it is practical.

Consider an effective action

$S_{5}=\int_{\mathcal{M}_{5}} d^{3+2} \xi \sqrt{-g}\left[R-\sum_{j=1}^{p} \eta_{j}\left(\partial \phi_{j}\right)^{2}-V\left(\left\{\phi_{j}\right\}\right)\right]$,

where $j=1 \cdots p$ indices the scalars $\phi_{i} ; \eta_{i}$ 's are the constant normalizations of the scalar kinetic terms, and $V$ is an arbitrary potential. We will be interested in the stability analysis of the black branes/holes in the theory (A1) with maximally symmetric 3-dimensional Schwarzschild horizons:

$$
d s_{5}^{2}=-c_{1}^{2} d t^{2}+c_{2}^{2} d X_{3, K}^{2}+c_{3}^{2} d r^{2}
$$

where $c_{i}=c_{i}(r), \phi_{j}=\phi_{j}(r)$ and

$d X_{3, K}^{2}=\left\{\begin{array}{llc}d x^{2} \equiv d x_{1}^{2}+d x_{2}^{2}+d x_{3}^{2}, & K=0, & \text { planar, } \\ d \Omega_{(3)}^{2}, & K>0, & \text { spherical, } \\ d H_{(3)}^{2}, & K<0, & \text { hyperbolic. }\end{array}\right.$

Note that we do not fix $K=\{0, \pm 1\}$, but instead allow it to vary smoothly-this would allow for the interpolation of the quasinormal spectra between different maximally symmetric horizons, notably between the planar and the spherical ones. A useful way to explicitly parametrize $X_{3, K}$ and a metric on it is as follows: $X_{3, K}=$ $\left(x_{1} \equiv x, x_{2} \equiv y, x_{3} \equiv z\right)$, and

\footnotetext{
${ }^{9}$ The motivation is to verify and fix typos in the original work [18], and to prepare the stage for the stability analysis of the black holes on the conifold with fluxes [38].
}

$d X_{3, K}^{2}=\frac{d x^{2}}{\left(1-K x^{2}\right)}+\left(1-K x^{2}\right)\left[\frac{d y^{2}}{\left(1-K y^{2}\right)}+\left(1-K y^{2}\right) d z^{2}\right]$.

From (A1) we obtain the following second order equations of motion $\left({ }^{\prime} \equiv \frac{d}{d r}\right.$ and $\left.\partial_{j} \equiv \frac{\partial}{\partial \phi_{j}}\right)$

$$
0=c_{1}^{\prime \prime}+c_{1}^{\prime}\left[\ln \frac{c_{2}^{3}}{c_{3}}\right]^{\prime}+\frac{c_{3}^{2} c_{1}}{3} V
$$

$$
\begin{gathered}
0=c_{2}^{\prime \prime}+c_{2}^{\prime}\left[\ln \frac{c_{1} c_{2}^{2}}{c_{3}}\right]^{\prime}+\frac{c_{2} c_{3}^{2}}{3}\left(V-\frac{6 K}{c_{2}^{2}}\right), \\
0=\phi_{j}^{\prime \prime}+\phi_{j}^{\prime}\left[\ln \frac{c_{1} c_{2}^{3}}{c_{3}}\right]^{\prime}-\frac{c_{3}^{2}}{2 \eta_{j}} \partial_{j} V,
\end{gathered}
$$

and the first order constraint ${ }^{10}$

$0=\sum_{j=1}^{p} \eta_{j}\left(\phi_{j}^{\prime}\right)^{2}-\left[\ln c_{2}^{3}\right]^{\prime}\left[\ln \left(c_{1}^{2} c_{2}^{2}\right)\right]^{\prime}+c_{3}^{2}\left(\frac{6 K}{c_{2}^{2}}-V\right)$.

We organize all the gauge invariant fluctuations into three sets of master scalars of different helicity $h$ :

(i) the helicity $h=2$ set, $\left\{\Phi_{2}^{(2)}\right\}$;

(ii) the helicity $h=1$ set, $\left\{\Phi_{2}^{(1)}\right\}$;

(iii) the helicity $h=0$ set, $\left\{\Phi_{2}^{(0)}, \Phi_{(0, j)}^{(0)}\right\}, j=1 \cdots p$. Any master scalar $\Phi_{s}^{(h)}(s=2$ or $s=(0, j)$ and $h=$ $\{0,1,2\})$ is assumed to have the following dependence:

$$
\Phi_{s}^{(h)}(\xi)=F_{s}^{(h)}(t, r) S\left(X_{3, K}\right),
$$

where $S\left(X_{3, K}\right)$ is a scalar eigenfunction of the Laplacian $\Delta_{K}$ on (A4) with an eigenvalue $k^{2}$ :

$$
\Delta_{K} S+k^{2} S=0 .
$$

In this work we will be concerned with planar $(K=0)$ or spherical $(K>0)$ horizons. In the former case, $k^{2} \in$ $[0,+\infty)$ and in the latter case

$$
k^{2}=K \ell(\ell+2) \quad \text { with } \quad \ell \in \mathbb{Z}_{+} .
$$

Each of the master scalars satisfies a coupled master equation of the form

$$
\square \Phi_{s}^{(h)}-W_{s, s^{\prime}}^{(h)}(r) \Phi_{s^{\prime}}^{(h)}=0,
$$

where $\square$ is the wave operator on the full $D=5$ metric (A2), and the symmetric potential matrix,

\footnotetext{
${ }^{10}$ We verified that (A8) is consistent with (A5)-(A7).
} 


$$
W_{s, s^{\prime}}^{(h)}=W_{s^{\prime}, s}^{(h)},
$$

couples master scalars in a given helicity set.

We now present results for potentials $W_{s, s^{\prime}}^{(h)}$ in different helicity sectors, as well as relations between the master scalars and a specific set of gauge invariant fluctuations in that sector. We refer the reader to [18] for a detailed discussion exactly how the gauge invariant fluctuations are constructed. ${ }^{11}$

\section{Helicity $h=0$ sector}

The relation between the gauge invariant fluctuations in the scalar sector

$$
\left\{\boldsymbol{\varphi}_{j}, \mathfrak{h}_{t r}, \mathfrak{h}_{r r}, \mathfrak{h}_{x r}, \mathfrak{h}_{t t}\right\},
$$

and the master scalars

$$
\left\{F_{(0, j)}^{(0)}, F_{2}^{(0)}\right\},
$$

all being functions of $(t, r)$, is as follows:

$$
\begin{gathered}
\boldsymbol{\varphi}_{j}=-\frac{1}{\sqrt{2 \eta_{j}}} F_{(0, j)}^{(0)}+\frac{\sqrt{3} k c_{2} \phi_{j}^{\prime}}{6 \tilde{k} c_{2}^{\prime}} F_{2}^{(0)}, \\
\mathfrak{h}_{r r}=\frac{c_{2} c_{3}^{2}\left(c_{2}^{\prime} c_{1}-c_{1}^{\prime} c_{2}\right)}{D} \sum_{j=1}^{p}\left\{\sqrt{2 \eta_{i}} \phi_{j}^{\prime} F_{(0, j)}^{(0)}\right\}+\left(\frac{c_{2}^{2} c_{3}^{2} k}{3 \sqrt{3}\left(c_{2}^{\prime}\right)^{2} \tilde{k}} \sum_{j=1}^{p}\left\{\eta_{j}\left(\phi_{j}^{\prime}\right)^{2}\right\}-\frac{c_{2} c_{3}^{2} c_{1}^{\prime} k}{\sqrt{3} c_{1} c_{2}^{\prime} \tilde{k}}-\frac{2 \sqrt{3} c_{1} c_{3}^{4} K k}{D \tilde{k}}\right. \\
\left.-\frac{k \sqrt{3} c_{3}^{2}\left(\left(c_{2}^{\prime}\right)^{2} c_{1}-c_{3}^{2} c_{1} k^{2}-c_{1}^{\prime} c_{2}^{\prime} c_{2}\right)}{\tilde{k} D}\right) F_{2}^{(0)}+\frac{c_{2} c_{3}^{2} k}{\sqrt{3} c_{2}^{\prime} \tilde{k}} \partial_{r} F_{2}^{(0)}, \\
\mathfrak{h}_{x r}=\frac{c_{2}^{2} c_{3}^{2} c_{1}}{D} \sum_{j=1}^{p}\left\{\sqrt{\frac{\eta_{j}}{2}} \phi_{j}^{\prime} F_{(0, j)}^{(0)}\right\}+\left(-\frac{3 \sqrt{3} c_{1} c_{2} c_{2}^{\prime} c_{3}^{2} K}{k \tilde{k} D}+\frac{c_{2} c_{3}^{2} k}{2 \sqrt{3} c_{2}^{\prime} D \tilde{k}}\left(c_{3}^{2} c_{1} k^{2}+3\left(c_{2}^{\prime}\right)^{2} c_{1}+3 c_{1}^{\prime} c_{2}^{\prime} c_{2}\right)\right) F_{2}^{(0)} \\
+\frac{\sqrt{3} c_{2}^{2}}{2 k \tilde{k}} \partial_{r} F_{2}^{(0)},
\end{gathered}
$$

where we set

$$
\tilde{k} \equiv \sqrt{k^{2}-3 K}, \quad D \equiv c_{3}^{2} c_{1} k^{2}-3\left(c_{2}^{\prime}\right)^{2} c_{1}+3 c_{1}^{\prime} c_{2}^{\prime} c_{2} .
$$

Additionally,

$$
\begin{gathered}
\mathfrak{h}_{t t}=\frac{2 c_{1}^{2}}{c_{3}^{2}}\left(\ln \frac{c_{3}}{c_{1} c_{2}}\right)^{\prime} \mathfrak{h}_{x r}-\frac{2 c_{1}^{2}}{c_{3}^{2}} \partial_{r} \mathfrak{h}_{x r}+\frac{c_{1}^{2}}{c_{3}^{2}} \mathfrak{h}_{r r}, \\
\mathfrak{h}_{t r}=\frac{4 c_{2}^{2}}{k^{2}} \sum_{j=1}^{p}\left\{\eta_{j} \phi_{j}^{\prime} \partial_{t} \boldsymbol{\varphi}_{j}\right\}+2 \partial_{t} \mathfrak{h}_{x r}-\frac{6 c_{2}^{\prime} c_{2}}{c_{3}^{2} k^{2}} \partial_{t} \mathfrak{h}_{r r} .
\end{gathered}
$$

The master scalars $\Phi_{s}^{(0)}$ satisfy (A12) with the potentials:

$$
\begin{aligned}
W_{2,2}^{(0)}= & \frac{4}{3 D^{2} c_{2}^{2}}\left(c_{2}^{2} c_{1}^{2} c_{3}^{2} k^{2} \tilde{k}^{2} \sum_{n=1}^{p}\left\{\eta_{n}\left(\phi_{n}^{\prime}\right)^{2}\right\}+\left(k^{2}-6 K\right) D^{2}-9 \tilde{k}^{2} c_{1}^{\prime} c_{2}^{\prime} c_{2} D\right. \\
& \left.-3 \tilde{k}^{2}\left(k^{2}-2 K\right) c_{1} c_{3}^{2} D+2 \tilde{k}^{4} c_{1} c_{3}^{2}\left(c_{3}^{2} k^{2} c_{1}+3 c_{1}^{\prime} c_{2}^{\prime} c_{2}\right)\right),
\end{aligned}
$$

\footnotetext{
${ }^{11}$ In a holographic setting gauge invariant fluctuations in Einstein-scalar theories were used for the first time in [43].
} 


$$
\begin{aligned}
W_{2,(0, i)}^{(0)}= & -\frac{\tilde{k} k c_{3}^{2} c_{1}}{D} \sqrt{\frac{2}{3 \eta_{i}}} \partial_{i} V-\frac{\tilde{k} k c_{2} c_{1}\left(c_{1}^{\prime} c_{2}-c_{2}^{\prime} c_{1}\right)}{D^{2}} \sqrt{\frac{8 \eta_{i}}{3}} \phi_{i}^{\prime} \sum_{n=1}^{p}\left\{\eta_{n}\left(\phi_{n}^{\prime}\right)^{2}\right\} \\
& +\frac{\tilde{k} k c_{1} c_{2}^{\prime}\left(D-c_{3}^{2} k^{2} c_{1}\right)}{c_{2} D^{2}} \sqrt{\frac{8 \eta_{i}}{3}} \phi_{i}^{\prime}+\frac{\tilde{k} k c_{1}^{2} c_{3}^{2} c_{2}^{\prime}\left(k^{2}-2 K\right)}{D^{2} c_{2}} \sqrt{24 \eta_{i}} \phi_{i}^{\prime}, \\
W_{(0, i),(0, j)}^{(0)}= & \frac{1}{2 \sqrt{\eta_{i}} \sqrt{\eta_{j}}} \partial_{i} \partial_{j} V-\frac{c_{2}\left(c_{2}^{\prime} c_{1}-c_{1}^{\prime} c_{2}\right)}{D}\left(\sqrt{\frac{\eta_{j}}{\eta_{i}}} \phi_{j}^{\prime} \partial_{i} V+\sqrt{\frac{\eta_{i}}{\eta_{j}}} \phi_{i}^{\prime} \partial_{j} V\right) \\
& +\frac{2 c_{2}^{2}\left(c_{2}^{\prime} c_{1}-c_{1}^{\prime} c_{2}\right)^{2}}{c_{3}^{2} D^{2}} \sqrt{\eta_{i}} \phi_{i}^{\prime} \sqrt{\eta_{j}} \phi_{j}^{\prime} \sum_{n=1}^{p}\left\{\eta_{n}\left(\phi_{n}^{\prime}\right)^{2}\right\}+\frac{\sqrt{\eta_{i}} \phi_{i}^{\prime} \sqrt{\eta_{j}}}{D^{2}}\left(2 c_{1}^{2} c_{3}^{2} k^{2}\left(k^{2}-2 K\right)\right. \\
& \left.+2 k^{2}\left(c_{2}^{\prime} c_{1}+2 c_{1}^{\prime} c_{2}\right)\left(c_{2}^{\prime} c_{1}-c_{1}^{\prime} c_{2}\right)-\frac{12 c_{2}^{\prime}\left(c_{2}^{\prime} c_{1}+c_{1}^{\prime} c_{2}\right)\left(c_{2}^{\prime} c_{1}-c_{1}^{\prime} c_{2}\right)^{2}}{c_{3}^{2} c_{1}}\right),
\end{aligned}
$$

where $\tilde{k}$ and $D$ are defined in (A19).

Notice that for $K>0$, the relations (A17) and (A18) between the master scalars and the gauge invariant fluctuations are singular for $\ell=0$ and $\ell=1$. This is so because in these cases the dynamical degrees of freedom are those of the scalars only. These cases must be treated separately [18].

\section{a. $\ell=0$}

For $\ell=0$, there are no $h_{t x}, h_{x r}$ and $h_{-}$components of the metric perturbations; furthermore, the gauge transformations can be used to set metric components $h_{t r}=0$ and $h_{+}=0$. Thus, we are left with perturbations

$$
\left\{\boldsymbol{\varphi}_{j}, h_{t t}, h_{r r}\right\}
$$

We find ${ }^{12}$

$$
\begin{gathered}
h_{r r}=\frac{2 c_{3}^{2} c_{2}}{3 c_{2}^{\prime}} \sum_{i=1}^{p}\left\{\eta_{i} \phi_{i}^{\prime} \boldsymbol{\varphi}_{i}\right\}, \quad \text { (A25) } \\
c_{1}^{2} \partial_{r}\left(\frac{h_{t t}}{c_{1}^{2}}\right)=-\frac{2 c_{1}^{2} c_{2}}{3 c_{2}^{\prime}} \sum_{i=1}^{p}\left\{\eta_{i} \phi_{i}^{\prime} \partial_{r} \boldsymbol{\varphi}_{i}\right\}+\frac{c_{1}^{2} c_{3}^{2} c_{2}}{3 c_{2}^{\prime}} \sum_{i=1}^{p}\left\{\partial_{i} V \boldsymbol{\varphi}_{i}\right\} \\
+\frac{2 c_{1}^{2} c_{2}^{2}}{9\left(c_{2}^{\prime}\right)^{2}} \sum_{j=1}^{p}\left\{\eta_{j}\left(\phi_{j}^{\prime}\right)^{2}\right\} \sum_{i=1}^{p}\left\{\eta_{i} \phi_{i}^{\prime} \boldsymbol{\varphi}_{i}\right\} \\
-\frac{4 c_{1}\left(c_{1} c_{2}^{\prime}+c_{2} c_{1}^{\prime}\right)}{3 c_{2}^{\prime}} \sum_{i=1}^{p}\left\{\eta_{i} \phi_{i}^{\prime} \boldsymbol{\varphi}_{i}\right\} .
\end{gathered}
$$

Introducing the master scalars as

\footnotetext{
${ }^{12}$ As pointed out in [18], here, as well as for $\ell=1$, there is also a certain inhomogeneous piece in the master equations. This piece must be set to zero to study fluctuations in a fixed-mass black hole background.
}

$$
\boldsymbol{\varphi}_{j}=-\frac{1}{\sqrt{2 \eta_{j}}} F_{(0, j)}^{(0)}
$$

we obtain master equations for $\Phi_{(0, j)}^{(0)}$ with $W_{(0, i),(0, j)}^{(0)}$ given formally by (A23) in the limit $k \rightarrow 0$.

\section{b. $\ell=1$}

For $\ell=1$ there is no $h_{-}$component of the metric fluctuations. We can use gauge transformations to set metric components $h_{t x}=0$ and $h_{+}=0$. Thus, are left with the (gauge variant) perturbations

$$
\left\{\boldsymbol{\varphi}_{j}, h_{t r}, h_{r r}, h_{x r}, h_{t t}\right\} .
$$

Note that the scalar eigenfunction for $\ell=1$ is explicitly

$$
S\left(X_{3, K}\right)=x \sqrt{K} .
$$

From the fluctuation equations of motion we find (compare with (A20))

$$
\begin{aligned}
& h_{t t}=\frac{2 c_{1}^{2}}{c_{3}^{2}}\left(\ln \frac{c_{3}}{c_{1} c_{2}}\right)^{\prime} h_{x r}-\frac{2 c_{1}^{2}}{c_{3}^{2}} \partial_{r} h_{x r}+\frac{c_{1}^{2}}{c_{3}^{2}} h_{r r}, \\
& h_{t r}=\frac{4 c_{2}^{2}}{k^{2}} \sum_{j=1}^{p}\left\{\eta_{j} \phi_{j}^{\prime} \partial_{t} \boldsymbol{\varphi}_{j}\right\}+2 \partial_{t} h_{x r}-\frac{6 c_{2}^{\prime} c_{2}}{c_{3}^{2} k^{2}} \partial_{t} h_{r r} .
\end{aligned}
$$

The remaining fluctuations can be expressed through the master scalars (A15) as

$$
\boldsymbol{\varphi}_{j}=-\frac{1}{\sqrt{2 \eta_{j}}} F_{(0, j)}^{(0)}+\frac{g c_{2} \phi_{j}^{\prime}}{c_{2}^{\prime}} F_{2}^{(0)},
$$




$$
\begin{aligned}
h_{r r}= & \frac{c_{2} c_{3}^{2}\left(c_{2}^{\prime} c_{1}-c_{1}^{\prime} c_{2}\right)}{D} \sum_{j=1}^{p}\left\{\sqrt{2 \eta_{i}} \phi_{j}^{\prime} F_{(0, j)}^{(0)}\right\}+2 g\left(\frac{c_{2}^{2} c_{3}^{2}}{3\left(c_{2}^{\prime}\right)^{2}} \sum_{j=1}^{p}\left\{\eta_{j}\left(\phi_{j}^{\prime}\right)^{2}\right\}\right. \\
& \left.-\frac{c_{2} c_{3}^{2} c_{1}^{\prime}}{c_{1} c_{2}^{\prime}}-\frac{6 c_{1} c_{3}^{4} K}{D}-\frac{3 c_{3}^{2}\left(\left(c_{2}^{\prime}\right)^{2} c_{1}-c_{3}^{2} c_{1} k^{2}-c_{1}^{\prime} c_{2}^{\prime} c_{2}\right)}{D}\right) F_{2}^{(0)}+2 g \frac{c_{2} c_{3}^{2}}{c_{2}^{\prime}} \partial_{r} F_{2}^{(0)}, \\
h_{x r}= & \frac{c_{2}^{2} c_{3}^{2} c_{1}}{D} \sum_{j=1}^{p}\left\{\sqrt{\frac{\eta_{j}}{2}} \phi_{j}^{\prime} F_{(0, j)}^{(0)}\right\}+2 g\left(-\frac{9 c_{1} c_{2} c_{2}^{\prime} c_{3}^{2} K}{k^{2} D}+\frac{c_{2} c_{3}^{2}}{2 c_{2}^{\prime} D}\left(c_{3}^{2} c_{1} k^{2}\right.\right. \\
& \left.\left.+3\left(c_{2}^{\prime}\right)^{2} c_{1}+3 c_{1}^{\prime} c_{2}^{\prime} c_{2}\right)\right) F_{2}^{(0)}+g \frac{3 c_{2}^{2}}{k^{2}} \partial_{r} F_{2}^{(0)},
\end{aligned}
$$

where $D$ is given by (A19), and $g$ is an arbitrary constant parameter of the unfixed gauge transformation [18]. Note that (A31), (A32) and (A33) are equivalent to (A31), (A17) and (A18) up to replacement

$$
2 g \leftrightarrow \frac{k}{\sqrt{3} \tilde{k}} .
$$

The only physical master equations are those for the scalars $\Phi_{(0, j)}^{(0)}$ (in these equations there is a decoupling of the "gauge" master scalar $\Phi_{2}^{(0)}$ ), with the relevant potentials $W_{(0, i),(0, j)}^{(0)}$ obtained setting $\tilde{k}=0$ in (A23). The equation for the gauge master scalar can also be obtained from the general expressions valid for $\ell \geq 2$, provided we identify (compare (A16) and (A31)

$$
\left.\left.F_{2}^{(0)}\right|_{\ell \geq 2} \equiv 2 g \frac{\sqrt{3} \tilde{k}}{k} F_{2}^{(0)}\right|_{\ell=1}
$$

prior to taking the limit $\tilde{k} \rightarrow 0$. Because of (A35), this latter equation is necessarily singular in the limit $g \rightarrow 0$.

\section{Helicity $h=1$ sector}

The relation between the gauge invariant fluctuations in the vector sector

$$
\left\{\mathfrak{h}_{t z}, \mathfrak{h}_{z r}\right\},
$$

and the master scalar

$$
\left\{F_{2}^{(1)}\right\}
$$

all being functions of $(t, r)$, is as follows:

$$
\begin{aligned}
\mathfrak{h}_{t z} & =\frac{3 c_{2} c_{1} c_{2}^{\prime}}{\tilde{k} c_{3}} F_{2}^{(1)}+\frac{c_{2}^{2} c_{1}}{\tilde{k} c_{3}} \partial_{r} F_{2}^{(1)}, \\
\mathfrak{h}_{z r} & =\frac{c_{3} c_{2}^{2}}{\tilde{k} c_{1}} \partial_{t} F_{2}^{(1)},
\end{aligned}
$$

where we deliberately introduced a singularity at $\ell=1$ i.e., $\tilde{k}=0$ (A19), to highlight the fact that the fluctuations (A36) are gauge invariant only for $\ell \geq 1$ [18].

The master scalar $\Phi_{2}^{(1)}$ satisfies (A12) with the potential

$W_{2,2}^{(1)}=\frac{1}{c_{3}^{2}} \sum_{j=1}^{p}\left\{\eta_{j}\left(\phi_{j}^{\prime}\right)^{2}\right\}-\frac{3 K}{c_{2}^{2}}+\frac{3\left(c_{2}^{\prime}\right)^{2}}{c_{2}^{2} c_{3}^{2}}-\frac{6 c_{1}^{\prime} c_{2}^{\prime}}{c_{1} c_{2} c_{3}^{2}}$.

\section{a. $\ell=1$}

The case $\ell=1$ is special since in this case there are no dynamical degrees of freedom [18]. Indeed, here, there are no $h_{x z}$ component of the metric fluctuations, and the gauge can be fixed setting $h_{z r}=0$. The remaining metric component $h_{t z}$ satisfies

$$
\partial_{r}\left(\frac{h_{t z}(t, r)}{c_{2}^{2}}\right)=0
$$

which is normalizable only if it identically vanishes.

\section{Helicity $h=2$ sector}

The relation between a particular gauge invariant fluctuation $\mathfrak{h}_{y z}(t, r)$ and the master scalar $F_{2}^{(2)}(t, r)$ in the tensor sector is as follows

$$
\begin{aligned}
\delta d s_{5}^{2} & =\delta g_{y z}\left(t, r, X_{3, K}\right) d y d z=\mathfrak{h}_{y z}(t, r) \frac{S_{T}(x)}{1-K y^{2}} d y d z \\
& =c_{2}^{2} F_{2}^{(2)}(t, r) \frac{S_{T}(x)}{1-K y^{2}} d y d z,
\end{aligned}
$$

where $S_{T}(x)$ satisfies [18]

$\left(1-K x^{2}\right) S_{T}^{\prime \prime}=-K x S_{T}^{\prime}-\left(k^{2}+2 K\left(1+\frac{K x^{2}}{1-K x^{2}}\right)\right) S_{T}$.

The master scalar

$$
\Phi_{2}^{(2)}(\xi) \equiv F_{2}^{(2)}(t, r) S\left(X_{3, K}\right)
$$



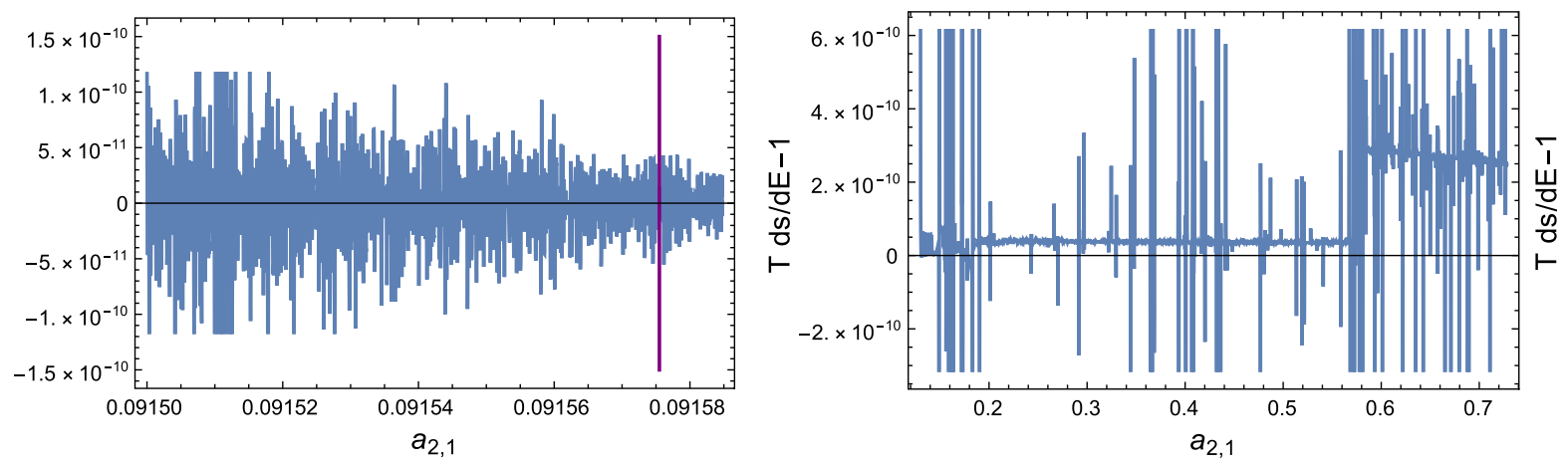

FIG. 16. Verification of the first law of thermodynamics (3.22) for the $\mathcal{N}=2^{*}$ black brane, $\frac{K}{m^{2}}=0$ (the left panel), and for a sample $\mathcal{N}=2^{*}$ black hole, $\frac{K}{m^{2}}=1$ (the right panel). The vertical purple line identifies the thermodynamically unstable state of interest (2.14), further used in the QNM analysis.

equation is universally that of the minimally coupled massless scalar

$$
\square \Phi_{2}^{(2)}=0,
$$

i.e.,

$$
W_{2,2}^{(2)} \equiv 0 .
$$

The universality of the $h=2$ sector at $K=0$ was emphasized originally in [44].

\section{Stability of $h=1$ and $h=2$ sectors}

It was argued in [18] that the QNMs of Einstein-scalar black holes/black branes, i.e., when $K \geq 0$, are stable in $h=2$ and $h=1$ helicity sectors.

Since $h=2$ sector is not directly sensitive to scalars of the effective action, it is obviously stable even for the more general effective actions (A1). The potential of the QNMs in the $h=1$ helicity sector explicitly depends on the scalars, see (A39). Nonetheless, the arguments of [18] can still be literally repeated, leading to the conclusion of the stability.

\section{APPENDIX B: NUMERICAL TESTS}

The work presented in this paper is numerical. It is imperative that we do as many tests as possible to confirm the reliability of the results. In the rest of this section we highlight a subset of the tests that we performed.

(1) The $\mathcal{N}=2^{*}$ black brane thermodynamics has been discussed previously in [22]. The previous work is done in a different parametrization of the background geometry, compare to the parameterization (3.7) used here. We recover ${ }^{13}$ the previous results.

(2) The hydrodynamics of $\mathcal{N}=2^{*}$ black brane was discussed earlier (again in a different parameterization) in $[9,23]$. Once again, we recover the previous results.

\footnotetext{
${ }^{13}$ Our current numerical algorithms are more precise.
}

(3) The $\mathrm{AdS}_{5}$-Schwarzschild $\Delta=2$ black brane spectrum at $\mathfrak{q}=0$ presented in Fig. 6 agrees with the results reported in [42], obtained using a completely different method.

(4) While we did not present the $\mathrm{AdS}_{5}$-Schwarzschild results for the $h=0$ sector graviton QNMs, ${ }^{14}$ obtained solving for the QNM spectrum from (3.39) at $\mathfrak{q}=\frac{1}{100}$, we confirmed that the results obtained are in agreement with $\Delta=4$ results reported in [42], obtained using a completely different method.

(5) We use the master field formalism [18], but we use finite difference rather than the spectral methods in computing the QNMs, as well as a different background geometry parameterization. We find agreement with the numerical result for the $\mathrm{AdS}_{5}$-Schwarzschild $h=0$ graviton QNM at $\mathfrak{q}=1$, see (3.41).

(6) In constructing $\mathcal{N}=2^{*}$ black brane and black hole geometries, we always verify the first law of thermodynamics (3.22). Typical results of such tests are shown in Fig. 16.

(7) $\mathcal{N}=2^{*}$ black hole background at temperature (2.14) and a given value of $\frac{K}{m^{2}}$ can be reached from $\mathrm{AdS}_{5}$-Schwarzschild horizon geometry in two ways: (a) we can keep $K=0$ and increase $\frac{m^{2}}{T^{2}}$ from zero to (2.14), followed by the increase of $\frac{K}{m^{2}}$ from zero to the value of interest;

(b) we can start with $\mathrm{AdS}_{5}$-Schwarzschild black hole at a corresponding value of $\frac{K}{T_{p}^{2}}$, and increase $\frac{m^{2}}{T^{2}}$ from zero to (2.14) — in both ways we land at an identical geometry.

(8) The hydrodynamic QNM of the $\mathcal{N}=2 *$ black brane in the thermodynamically unstable state (2.14) reported in Fig. 5 is in excellent agreement with the prediction (1.4), using the appropriate equilibrium transport coefficients (2.19). 
[1] L. D. Landau and E. M. Lifshitz, Statistical Physics, Part 1, Vol. 5 of Course of Theoretical Physics (ButterworthHeinemann, Oxford, 1980).

[2] J. M. Maldacena, The large N limit of superconformal field theories and supergravity, Int. J. Theor. Phys. 38, 1113 (1999).

[3] O. Aharony, S. S. Gubser, J. M. Maldacena, H. Ooguri, and Y. Oz, Large $\mathrm{N}$ field theories, string theory and gravity, Phys. Rep. 323, 183 (2000).

[4] E. Berti, V. Cardoso, and A. O. Starinets, Quasinormal modes of black holes and black branes, Classical Quant. Grav. 26, 163001 (2009).

[5] S. S. Gubser and I. Mitra, Instability of charged black holes in anti-de Sitter space, Clay Math. Proc. 1, 221 (2002).

[6] S. S. Gubser and I. Mitra, The evolution of unstable black holes in anti-de Sitter space, J. High Energy Phys. 08 (2001) 018.

[7] A. Ishibashi and H. Kodama, Stability of higher dimensional Schwarzschild black holes, Prog. Theor. Phys. 110, 901 (2003).

[8] A. Buchel, A holographic perspective on Gubser-Mitra conjecture, Nucl. Phys. B731, 109 (2005).

[9] A. Buchel and C. Pagnutti, Bulk viscosity of $N=2^{*}$ plasma, Nucl. Phys. B816, 62 (2009).

[10] A. Buchel, Critical phenomena in $N=4$ SYM plasma, Nucl. Phys. B841, 59 (2010).

[11] A. Buchel and C. Pagnutti, Correlated stability conjecture revisited, Phys. Lett. B 697, 168 (2011).

[12] A. Buchel and A. Patrushev, Can the correlated stability conjecture be saved?, J. High Energy Phys. 06 (2011) 090.

[13] A. Buchel, Thermal order in holographic CFTs and no-hair theorem violation in black branes, Nucl. Phys. B967, 115425 (2021).

[14] A. Buchel, SUGRA/strings like to be bald, Phys. Lett. B 814, 136111 (2021).

[15] A. Buchel, Fate of the conformal order, Phys. Rev. D 103, 026008 (2021).

[16] A. Buchel, Klebanov-Strassler black hole, J. High Energy Phys. 01 (2019) 207.

[17] H. Kodama and A. Ishibashi, A master equation for gravitational perturbations of maximally symmetric black holes in higher dimensions, Prog. Theor. Phys. 110, 701 (2003).

[18] A. Jansen, A. Rostworowski, and M. Rutkowski, Master equations and stability of Einstein-Maxwell-scalar black holes, J. High Energy Phys. 12 (2019) 036.

[19] K. Pilch and N. P. Warner, $N=1$ supersymmetric renormalization group flows from IIB supergravity, Adv. Theor. Math. Phys. 4, 627 (2000).

[20] A. Buchel, A. W. Peet, and J. Polchinski, Gauge dual and noncommutative extension of an $N=2$ supergravity solution, Phys. Rev. D 63, 044009 (2001).

[21] N. J. Evans, C. V. Johnson, and M. Petrini, The enhancon and $N=2$ gauge theory: Gravity RG flows, J. High Energy Phys. 10 (2000) 022.

[22] A. Buchel, S. Deakin, P. Kerner, and J. T. Liu, Thermodynamics of the $N=2^{*}$ strongly coupled plasma, Nucl. Phys. B784, 72 (2007).
[23] A. Buchel, Bulk viscosity of gauge theory plasma at strong coupling, Phys. Lett. B 663, 286 (2008).

[24] A. Buchel, Relaxation time of non-conformal plasma, Phys. Lett. B 681, 200 (2009).

[25] A. Buchel, Compactifications of the $N=2^{*}$ flow, Phys. Lett. B 570, 89 (2003).

[26] A. Buchel, Black hole spectra in holography: Consequences for equilibration of dual gauge theories, Nucl. Phys. B896, 587 (2015).

[27] HoloTube 2021: Tabilization of the Extended Horizons edited by A. Buchel, https://www.youtube.com/watch?v= HZkTkVvPxOE.

[28] A. Buchel, J. G. Russo, and K. Zarembo, Rigorous test of non-conformal holography: Wilson loops in $N=2^{*}$ theory, J. High Energy Phys. 03 (2013) 062.

[29] N. Bobev, H. Elvang, D. Z. Freedman, and S. S. Pufu, Holography for $N=2^{*}$ on $S^{4}$, J. High Energy Phys. 07 (2014) 001.

[30] N. Bobev, F. F. Gautason, and J. Van Muiden, Precision holography for $\mathcal{N}=2^{*}$ on $S^{4}$ from type IIB supergravity, J. High Energy Phys. 04 (2018) 148.

[31] A. Buchel (to be published).

[32] R. Baier, P. Romatschke, D. T. Son, A. O. Starinets, and M. A. Stephanov, Relativistic viscous hydrodynamics, conformal invariance, and holography, J. High Energy Phys. 04 (2008) 100.

[33] A. Buchel and C. Pagnutti, Critical phenomena in $N=2^{*}$ plasma, Phys. Rev. D 83, 046004 (2011).

[34] A. Buchel, On Eling-Oz formula for the holographic bulk viscosity, J. High Energy Phys. 05 (2011) 065.

[35] A. Buchel and J. T. Liu, Universality of the Shear Viscosity in Supergravity, Phys. Rev. Lett. 93, 090602 (2004).

[36] P. Kovtun, D. T. Son, and A. O. Starinets, Viscosity in Strongly Interacting Quantum Field Theories from Black Hole Physics, Phys. Rev. Lett. 94, 111601 (2005).

[37] C. Hoyos, S. Paik, and L. G. Yaffe, Screening in strongly coupled $N=2^{*}$ supersymmetric Yang-Mills plasma, J. High Energy Phys. 10 (2011) 062.

[38] A. Buchel, A bestiary of black holes on the conifold with fluxes, arXiv:2103.15188.

[39] A. Buchel, $N=2^{*}$ hydrodynamics, Nucl. Phys. B708, 451 (2005).

[40] A. Buchel, L. Lehner, and R. C. Myers, Thermal quenches in $N=2^{*}$ plasmas, J. High Energy Phys. 08 (2012) 049.

[41] O. Aharony, A. Buchel, and P. Kerner, The black hole in the throat: Thermodynamics of strongly coupled cascading gauge theories, Phys. Rev. D 76, 086005 (2007).

[42] A. Nunez and A. O. Starinets, AdS/CFT correspondence, quasinormal modes, and thermal correlators in $N=4 \mathrm{SYM}$, Phys. Rev. D 67, 124013 (2003).

[43] P. Benincasa, A. Buchel, and A. O. Starinets, Sound waves in strongly coupled non-conformal gauge theory plasma, Nucl. Phys. B733, 160 (2006).

[44] A. Buchel, On universality of stress-energy tensor correlation functions in supergravity, Phys. Lett. B 609, 392 (2005). 